Historic, archived document

Do not assume content reflects current scientific knowledge, policies, or practices. 

Semi-Annual

Wholesale Trade

List

Fall 1920

For Nurserymen Only

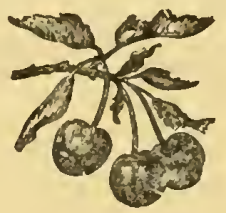

1870---Fiftieth Anniversary---1920

Shenandoah Nurseries

D. S. LAKE, President

Shenandoah, - - Iowa 


\section{For Your Information}

To those who are not acquainted with our concern, would say that this business was established in 1870 , and is still under the same management.

We are located in Southwestern Iowa, fifty miles southeast of Omaha, Nebraska. The soil here is especially adapted to the growing of a general line of nursery stock. The railroad facilities are excellent. We are on the Wabash, Ch'cego, Burlington \& Quincy, Keckuk \& Western railroads. We take pleasure in showing visitors over our grounds at any time.

\section{TERMS OF SAIE.}

SHIPPING-With the exception of Evergreens and some heavy shade trees we are in position to ship assorted orders all through the winter, and can, as a rule, get refrigerator service, although we do not guarantee this. Can begin to dig trees in this latitude usually about October 1. Parties ordering goods should indicate whether they wish them sent by freight, express or parcel post, also route. In the absence of these instructions, we forward them to the best of our judgment, but in no case will we assume responsibility after consignment to purchaser. When losses occur by delays or other causes of whatever nature while goods are in transit, make claim AT ONCE on the forwarding companies. All shipments travel at the risk and cost of the purchiser.

TSRMS-Cash or security before shipment of goods, except with esta'lished firms, when, if unknown to us, satisfactory references will be required before goods are forwarded. Orders to be sent C. O. D. should be accompanied by one-fourth cash.

PRICES-Are limited to Fall 1920 and supersede all previous lists and ARE SUBJECT TO CHANGE WITHOUT NOTICE.

CAUTION-We accept all orders upon condition that they shall be void should any injury befall stock from frost, fire, hail storms or other causes over which we have no control.

CLAIMS-For any cause will receive consideration only when made within ten days after the arrival of goods. In no case will we entertain claims made after the goods have been once accepted.

PACKING-Prices cuntained herein are net; cost of boxing and baling will ba added. No charge is made for loading bulk cars.

GRADES - We grade carefully; in some cases by caliper, in others by height. When graded by both caliper and height, the caliper must govern.

CERTIFICATE OF INSPECTION-Will be furnished with each shipment.

GUARANTEE OF GENUINENESS-If any nursery stock, etc. proves untrue to description under which it was sold, we hold ourselves in readiness on proper proof, to replace all sucli nursery stock, etc., that may prove otlerwise or refund the amount paid, but we shall in no case be liable for any sum greater than the amount originally received for said nursery stock.

\section{BOXING AND PACKNNG CEARGES}

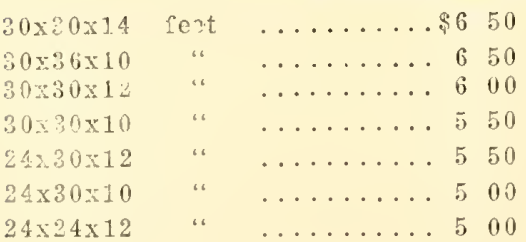

$24 \times 24 \times 10$ feet

$20 \times 20 \times 10$

$18 \times 18 \times 10$

$16 \times 16 \times 10$

$14 \times 14 \times 10$

$12 \times 12 \times 10$

$12 \times 12 \times 8$

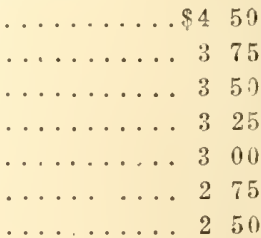




\section{FRUIT DEPARTMENT}

\section{APPLE}

Per 100

Per 1000

Siandard First Class, 4 1-2 to 6 feet, 11-16 inch and up...\$55 00

Siandard First Class. 4 to 5 feet, $5-8$ to 11-16 inch...... 4500

Standard First Class, 3 1-2 to 5 feet, 1-2 to 5-8 inch..... 3000

Alexanter

Arkansas Black

Aitumn Strawberry

Baldwin

Fam na

Ben Davis

I3elloni

I3lalk Jen I)avis

Delicious

lonininee

Durliess of Oldenlurg

Inulley

Early Harrest

1. (i, Russett

Fall Pippin

Famcuse

fiano

fiolden Sweet

rimes Golden

II ibernal

liuntsman

lisgram

Iow: Beauty

Iowa Blush

Innet

Tonatlan

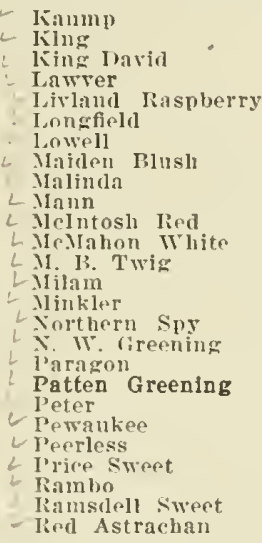

CRAB

\author{
Red Beitigheimer \\ Irol June \\ I. I. Freening \\ lioman Stem \\ linme Beanty \\ lioxbury liussett \\ Salom \\ Scott's Winter \\ Stark \\ - Stayman Winesap \\ Sweet June \\ LTolman Sweet \\ Iniversity \\ IV a gener \\ IValiridge \\ Wealthy \\ Wm. Early lied \\ Tillow Twig \\ Winesap \\ VI. W. Pearmain \\ Wisconsin kussett \\ IVolf liver \\ Yellow Pelle \\ Yellow Transparent \\ Vork Imperial
}

t.

\begin{tabular}{|c|c|c|}
\hline Standard Fi & $1-2$ to 6 feet, $11-16$ & up...\$55 00 \\
\hline Standard Fi & to 5 feet, $5-8$ to 11 & . 4500 \\
\hline Standard Fi & $1-2$ to 5 feet, $1-2$ to & $\ldots \ldots 30 \quad 00$ \\
\hline Standard Fi & to 4 feet, $3-8$ to $1-$ & $\ldots \ldots 2000$ \\
\hline $\begin{array}{l}\text { Alaska } \\
\text { Florénce } \\
\text { Feneral firant } \\
\text { Myslop } \\
\text { Martha }\end{array}$ & $\begin{array}{l}\text { Nlinnesota } \\
\text { Qmeen C. Cluster } \\
\text { Red Siberian } \\
\text { Shipli } \\
\text { Sylran Sweet }\end{array}$ & $\begin{array}{l}\text { - Transcendent } \\
\text { \& White Arctic } \\
\text { LWhitney } \\
\text { Yellow Siberian }\end{array}$ \\
\hline
\end{tabular}

Per 1000

$\$ 500 \quad 00$

$400 \quad 00$

25000

17500

\section{CHERRY-0n Mahaleb}

Per 100

Two Year, 4 to 6 feet, 11-16 inch and up.........\$75 00

Two Year, 3 1-2 to 5 feet, 9-16 to 11-16 inch.........6 6000

Two Year, 3 to 4 leet, $7-16$ to $9-16$ inch.............. 4500

Black Tartarian

Dyehouse

Early Rlehmond

English Morello

Gor. Wood

T.ambert

- lay Inuke

Iontmorency Iarge

Napoleon

bolivet

Ostheim

LWragg

Per 100

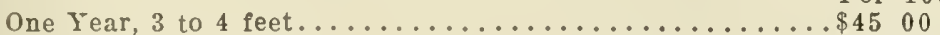

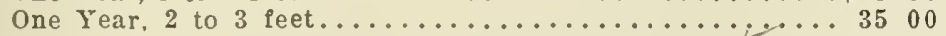

Early Rfchmond

Montmorency Isarge

\section{COMPASS CHERRY-PLUM-0n Native Roots}

One and Two Years, 11-16 inch and up.................. Per 100

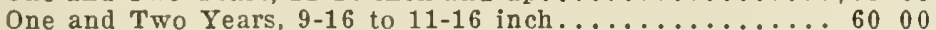

One and Two Years, $7-16$ to $9-16$ inch...........45 00

One and Two Years, $5-16$ to $7-16$ inch .............. 3000 


\section{QUINCE}

Per 100

Two Years, 4 to 5 feet, $5-8$ inch and $u p \ldots \ldots \ldots \ldots \ldots \ldots \ldots 0$

Two Years, 3 to 4 feet, $1-2$ to $5-8$ inch........... 6000

Two Years, 2 to 3 feet, $3-8$ to $1-2$ inch............ 4000

Champion

Orange

Meech's

Lieas Mammoth

\section{MUIBERRY}

Russian

6 to 8 ft 5 to $6 \mathrm{ft}+$ to $5 \mathrm{ft}: 3$ to $+\mathrm{ft}$

\section{PEACH}

Per 100

One Year, 5 to 6 feet, $11-16$ inch and up.........\$40 00

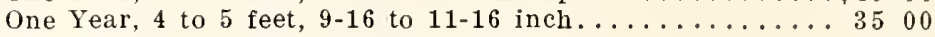

One Year, 3 to 4 feet, $7-16$ to $9-16$ inch............ 2800

One Year, 2 1-2 to $31-2$ feet, 5-16 to $7-16$ inch........20 00

Alexander

Carman

Champion

Chinese Cling

Cooledge lavorite

crawford Farly

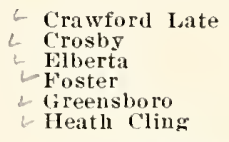

\section{APRIC0T-Budded}

Per 100

One and Two Year, 5 to 6 feet, 11-16 inch and up.....\$75 00

One and Two Year, 4 to 5 feet, $9-16$ to $11-16 \mathrm{inch} \ldots \ldots \ldots 6000$

One and Two Year, 3 to 4 feet, $7-16$ to $9-16$ inch $\ldots \ldots \ldots . . .4500$

One and Two Year, 2 1-2 to $31-2$ feet, $5-16$ to $7-16$ inch. 2500

One and Two Year, 2 to 3 feet................... 1500

Alexander

Tarly Golden

$L$ Moorpark
lioyal

Superb

IVilsou

Per 100

Russian, Own Roots, 5 to 6 feet, $11-16$ inch and up.....\$40 00 Russian, Own Roots, 4 to 5 feet, $9-16$ to $11-16$ inch...... 3000 Russian, Own Roots, 3 to 4 feet, $7-16$ to $9-16$ inch...... 2000 Russian, Own Roots, 2 1-2 to $31-2$ feet, $5-16$ to $7-16$ inch. . 1500 Russian, Own Roots, 2 to 3 feet............... 1000

\section{PEAR-Standard \\ (Budded on French Branched Seedlings)}

Per 100

Two Years, 5 feet and up, 11-16 inch and up........\$75 00

Two Years, 4 to 5 feet, $9-16$ to $11-16$ inch........... 6000

Two Years, $31-2$ to 4 1-2 feet, $7-16$ to $9-16$ inch...... 4500

Two Years, 2 1-2 to 3 1-2 feet, 5-16 to $7-16 \mathrm{inch} \ldots \ldots \ldots . .2500$

\section{Bartlett}

B. Bose

B. Clairgeau

Duchess d' Angouleme

Early Harvest clapp lavorite

Flemish Beauty
Carber
Kieffer
Loonce
Lawrence
Lossney

\section{PEAR-Dwarf}

Per 100

Two Years, $5-8$ inch and $u p \ldots \ldots \ldots \ldots \ldots \ldots \ldots \ldots \ldots \ldots \ldots \ldots$

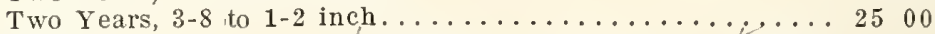

Bartlett

Clapp Favorite
Duchess d' Angouleme Garber

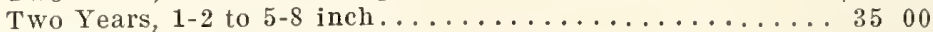

\section{Seckel}

Vermont Beauty

Worden Seckel $\cdots \ldots$

$\ldots \ldots$

$\ldots \ldots$

$\ldots \ldots$

$\ldots \ldots$

$\ldots \ldots$

$\ldots \ldots$

$\ldots \ldots$

$\ldots \ldots$

$\ldots \ldots$

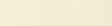

$\ldots \ldots$

$\ldots \ldots$

$\ldots \ldots$

$\ldots \ldots$

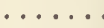

$\ldots \ldots$ 


\section{PLUM-0n Native Roots \\ (Americana and Chicasaw Varieties)}

One and Two Years, 5 to 6 feet, 11-16 inch and up..... Per 10 \& $\$ 75.00$

One and Two Years, 4 to 6 feet, 9-16 to 11-16 inch..... 6000

One and Two Years, 3 to 4 feet, $7-16$ to $9-16$ inch..... 4500

one and Two Years, 2 1-2 to 3 1-2 feet, 5-16 to 7-16 inch 3000

One and Two Years, 2 to 3 feet................... 2000

Brittlewood

Burwood

Cheney:

Desotia

Formst fiarden

Haminer

\author{
llawkeye \\ Ounaha \\ Pottawattamie \\ Quaker \\ Stodilara \\ Surprise
}

Terry

Weaver

Will cioose

Wolf

Wyaut

\section{PLUM-0n Native Roots \\ (Hansen's New Plums)}

One and Two Years, 5 to 6 feet, 11-16 inch and up... Per 100

One and Two Years, 4 to 5 feet, $9-16$ to $11-16$ inch..... 6000

One and Two Years, 3 to 4 feet, 7-16 to 9-16 inch.... 4500

One and Two Years, 2 1-2 to 3 1-2 feet, 5-16 to 7-16 inch 3000

one and Two Years, 2 to 3 feet............... 2000

Hanska

Clieresota

Opata

Sia Waneta

\section{PLUM-On Plum and Peach \\ (Japanese Varieties)}

One and Two Years, 5 to 6 feet, 11-16 inch and up.....

One and Two Years, 4 to 6 feet, 9-16 to 11-16 inch.....

One and Two Years, 3 to 4 feet, $7-16$ to 9-16 inch.....

One and Two Years, 2 1-2 to $31-2$ feet, 5-16 to 7-16 inch

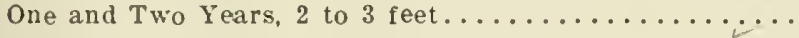

Abundance

Prunus Simoni

Wickson

Burbank

- Red June

Per 100

$\$ 70 \quad 00$

6000

4500

$\begin{array}{lll}30 & 00\end{array}$

$20 \quad 00$

\section{PLUM-0n Plum \\ (European Varieties)}

One and Two Years, 5 to 6 feet, 11-16 inch and up..... \$75 00

One and Two Years, 4 to 6 feet, $9-16$ to $11-16$ inch.... 60.00

One and Two Years, 3 to 4 feet, $7-16$ to $9-16$ inch..... 4500

One and Two Years, 2 1-2 to $31-2$ feet, 5-16 to 7-16 inch 3000

Bradshaw

Gueii

Imperial Gage

Liberty I'rume

\section{rombard}

Moore's Arctic

Pond seelling

reine Claude
Shipper's Iride

Siliropshire Darnsou

\section{G00SEBERRY}

2 Year. No. 1

\begin{tabular}{|c|c|c|c|c|}
\hline & Per 100 & & & Per 100 \\
\hline Carrie & $\$ 20 \quad 00$ & $\ldots \ldots$ & Houghton & $\$ 1800$ \\
\hline Champion & $20 \quad 00$ & $\ldots \ldots$ & Josselyn .... & 2200 \\
\hline Chautauqua . & 2500 & $\ldots \ldots$ & Pearl ...... & 2200 \\
\hline Downing .... & $20 \quad 00$ & $\ldots \ldots$ & & \\
\hline
\end{tabular}

1 Year, No. 1, or 2 Year, No. 2

Carrie ... \$15 00

Champion ... 1500

Per 100

Chautauqua . 2000

Houghton ... \$12 50

Downing ....

1500 


\section{STRAWBERRIES}

(Strawberries should be shipped by express)

Shipment after oct. 10

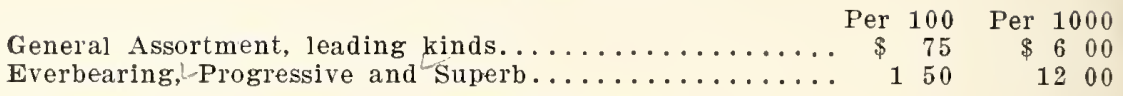

\section{GRAPE VINES}

2 Year, No. 1

\begin{tabular}{|c|c|c|c|c|c|c|}
\hline & & Per & 100 & Per 1000 & & Per 10 \\
\hline Agawam & $\ldots$ & $\$ 18$ & 00 & $\ldots \ldots$ & $\angle$ Lindley & $\$ 18 \quad 00$ \\
\hline Beta & $\ldots \ldots$ & 20 & 00 & $\cdots$ & L Moore's & \\
\hline Brighton & $\ldots$ & 20 & 00 & 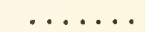 & Diamond & 18 \\
\hline Campbell & $\ldots$ & 22 & 00 & $\ldots \ldots$ & $\therefore$ Moore's Early & 22 \\
\hline Catawba & $\ldots$ & 18 & 00 &. & ¿ Niagara .... & 18 \\
\hline Champion & $\ldots$ & 16 & 00 & & \& Pocklington .. & 18 \\
\hline Clinton. & $\ldots \ldots$ & 16 & 00 & & Salem .... & 20 \\
\hline Concord & $\ldots$ & 16 & 00 & $150 \quad 00$ & Vergennes & 18 \\
\hline Delaware & $\ldots$ & 20 & 00 & $\ldots \ldots$ & Worden & 20 \\
\hline lvira & $\ldots \ldots$ & 18 & 00 & & Wyoming Red & 18 \\
\hline
\end{tabular}

Per 1000

1 Year, No. 1. or 2 Year. No. 2

Per 100 Per 1000

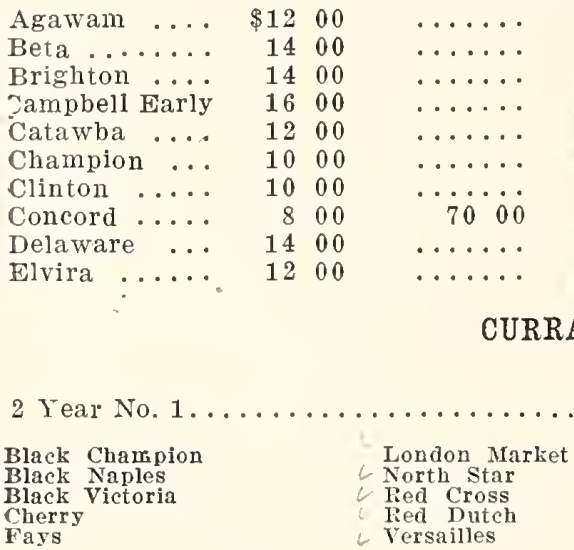

Agawam ....

Brighton ....

Campbell Early

Catawba ....

Champion ...

Clinton $\ldots .$.

Delaware ...

Elvira

\section{Lindley \\ Moore's \\ Diamond .. \\ Moore's Early \\ Niagara .... \\ Pocklington. \\ Salem ..... \\ Vergennes .. \\ Worden .... \\ Wyoming Red}

Per 100

$\$ 1200$

1200

1500

1200

1200

1400

1200

1400

1200

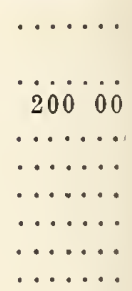

Per 1000

Per 100

Per 1000

$\$ 2250$

$\$ 200 \quad 00$

White Dutch

White Grape

IVilder

1 Year No. 1 ol 2 Year No.

$\$ 17 \quad 50$

$\$ 15000$

London Market

North Star

Fed Cross

Red Dutch

Versailles
Victoria Red

White Duteh

Wilder

Fays

Perfection, 2 Year, No. 1

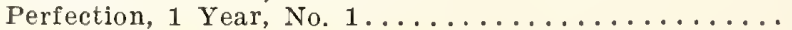

Tree Currants, Red and Black. 


\section{RASPBERRIES}

(Black Caps should be shipped by Express)

Black Pearl, Cumberland, Grege, Kansas and Plum Farmer,

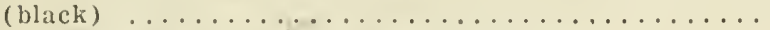

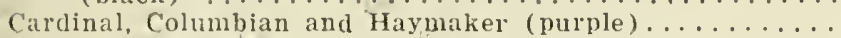

Cuthbert, King, Marlboro and Miller (red) ...........

St, Regis and Tierbert ( ted)

\section{BLACKBERRIES}

Per 100 Per 1000

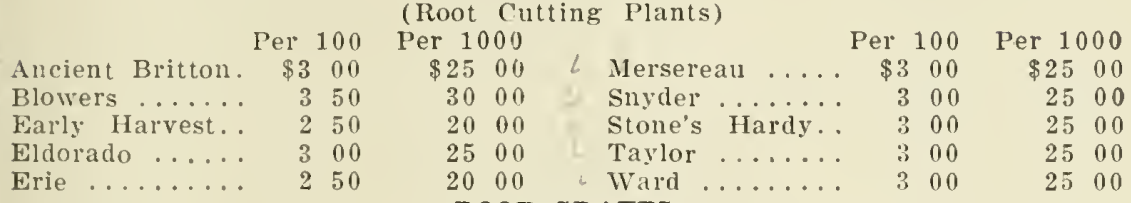

\section{ROOT GRAFTS}

$\begin{array}{ccccc}\$ 2 & 50 & & \$ 20 & 00 \\ 3 & 50 & & 30 & 00 \\ 2 & 50 & & 20 & 00 \\ 3 & 00 & & 25 & 00\end{array}$

We are in position to supply a good assortment of Piece or Whole Root Apple Grafts for Spring of 1921 planting. Send for list of varieties.

Prices on application.

\section{AMERICAN GROWN APPLE SEEDLINGS}

This has beell an ideal season for growing. Apple Seedlings as well as all other Nursery Stock.

Rains have been favorable and there have been no insects or diseases of any kind. At present our Seedlings promise to be excellent.

We can ship from Shenandoah, Iowa, Topeka or Rossville, Kansas. Early orders we can give car load rates to Rochester, N. Y., Winchester, Tenn., and some other points.

Write for particulars.

Order early.-Boxing extra.

Apple Seedlings, 3-16 inch and all up, straight or branched, at $\$ 2500$ per II Apple Seedlings. No. 2, 2-16 to 3-16 inch, straight or branched 1500 per $\mathbf{M}$ Apple Seedlings, No. 3................... 500 per MI

Apple Seedlings, No. $4 \ldots \ldots \ldots \ldots \ldots \ldots \ldots \ldots \ldots \ldots \ldots \ldots$ per $\mathbf{M}$

The No. 3 are suitable for light grafting, or lining out to bud.

\section{IMPORTED FRENCH FRUIT TREE STOCKS}

These prices are net at Shenandoah. Iowa. (Boxing extra.) We have a good supply of Frencl grown Apple, Pear, Mahaleb, Quince, etc. Ask for prices on any foreign stocks not mentioned in this list.

Early orders can be packed up in France and shipped direct from New York to customer. In this case, car load freight from New York to Shenandoah, Iowa, will be deducted.

Apple, $7-12 \mathrm{~mm} \ldots \ldots \ldots \ldots \ldots \ldots \ldots \ldots \ldots \ldots \ldots \ldots \ldots \ldots$ per $\mathbf{M}$

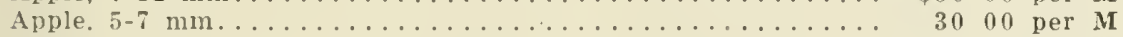

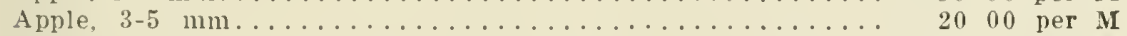

Pear, $7-12 \mathrm{~mm} \ldots \ldots \ldots \ldots \ldots \ldots \ldots \ldots \ldots \ldots \ldots \ldots \ldots \ldots \ldots \ldots$ per $80 \ldots \ldots$

Pear, $5-7 \mathrm{~mm} \ldots \ldots \ldots \ldots \ldots \ldots \ldots \ldots \ldots \ldots \ldots \ldots \ldots \ldots \ldots \ldots$. $60 \ldots \ldots$ per

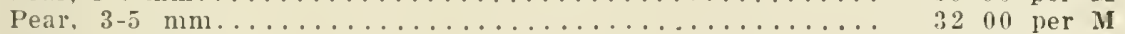

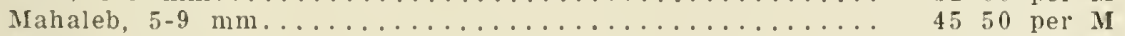

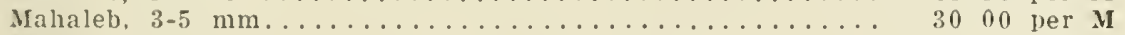

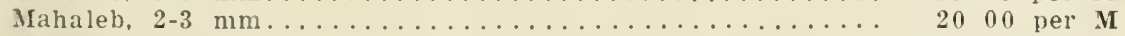

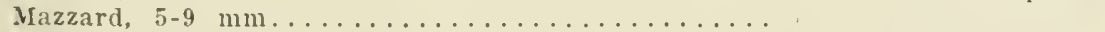

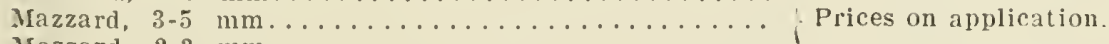

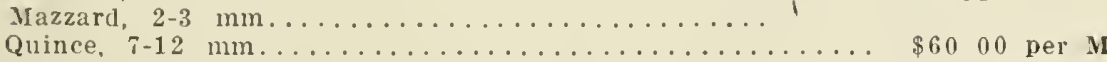

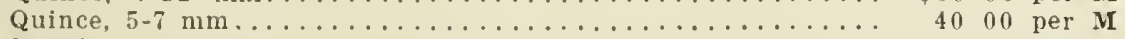

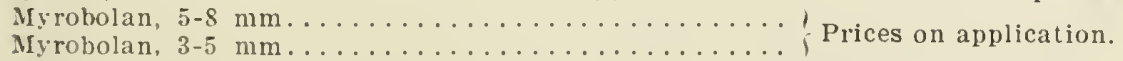




\section{DEWBERRIES}

Lucretia, Tip Plants.

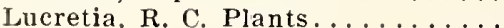

\section{ASPARAGUS}

Barr's Mammoth, Columbian, Conover's and Palmetto, 2 yr. Barr's Mammoth, Columbian, Conover's and Palmetto. 1 yr. MISCELLANEOUS

Horse Radish

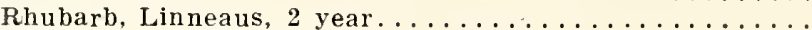

Rhubarb, Linneaus, 1 year.................

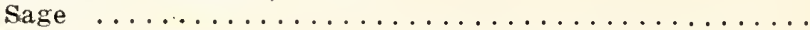

Strawberry-Raspberry

\section{FOREST TREE SEEDLINGS}

Ash, 3 to 4 feet.

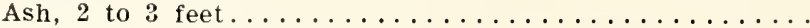

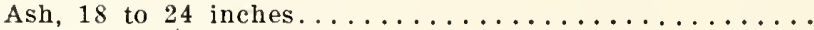

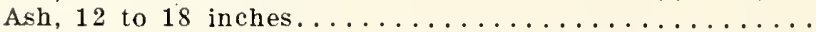

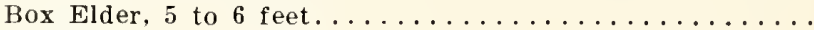

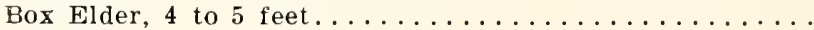

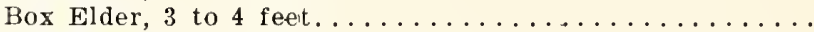

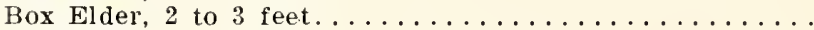

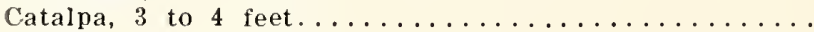

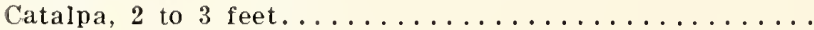

Catalpa, 18 to 24 inches.

Catalpa, 12 to 18 inches $\ldots \ldots \ldots \ldots \ldots \ldots \ldots \ldots$

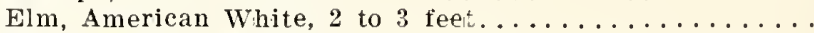

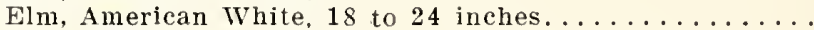

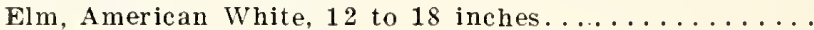

Locust, Black, 3 to 4 feet.................

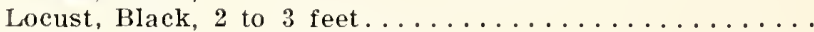

Locust, Black, 18 to 24 inehes...............

Locust, Black, 12 to 18 inches................

Locust, Honey, 2 to 3 feet................

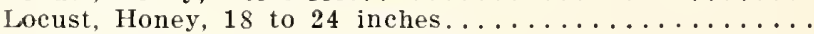

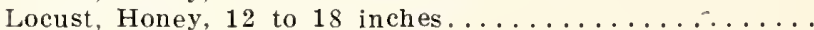

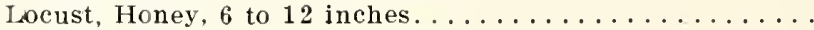

Maple, Silver Leaved, 3 to 4 feet..............

Maple, Silver Leaved, 2 to 3 feet..............

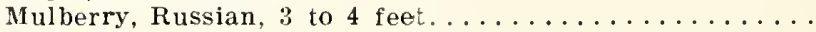

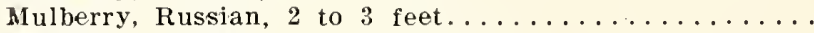

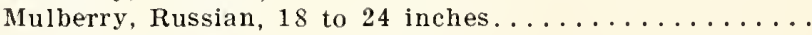

Mulberry, Russian, 12 to 18 inches.............

Olive, Russian, 18 to 24 inches...............

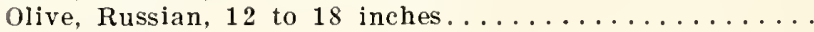

Olive. Russian, 6 to 12 inches................

Osage Orange, No. $1 \ldots \ldots \ldots \ldots \ldots \ldots \ldots \ldots \ldots$

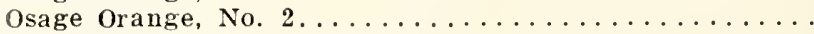

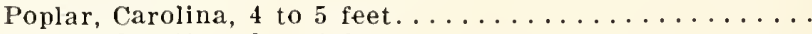

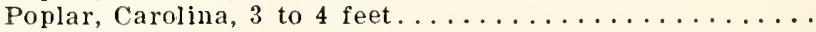

Poplar, Carolina, 2 to 3 feet...............

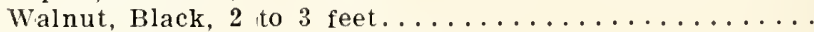

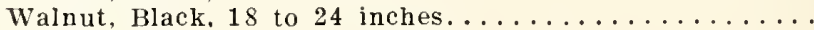

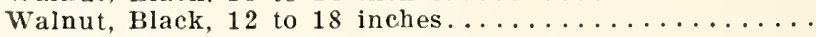

Willow, Russian Golden, 3 to 4 feet.............

Willow, Russian Golden, 2 to 3 feet.............

Willow, Russian Golden, 18 to 24 inches............

\section{Per 100 Per 1090 \\ $\$ 300$ \\ 350 \\ $\$ 25 \quad 00$ \\ $30 \quad 00$}

Per 100 Per 1000

125

85

1000

700

Per 100 Per 1000

$\begin{array}{llll}175 & 1500\end{array}$

$\begin{array}{llll}4 & 00 & 35 & 00\end{array}$

$\begin{array}{llll}3 & 00 & 25 & 00\end{array}$

$\begin{array}{ll}10 & 00\end{array}$

300

Per 100

$\$ 200$

100

75

60

$6 \quad 00$

350

250

160

140

$1 \quad 10$

85

175

150

100

200

150

125

100

150

125

100

Per 1001

$\$ 1500$

800

600

450

$50 \quad 00$

$30 \quad 00$

$20 \quad .00$

1400

1200

900

700

500

1500

1200

800

1600

1250

$10 \quad 00$

800

1250

$10 \quad 00$

800

$4 \quad 00$

2500

1750

2200

1500

1000

700

$\begin{array}{ll}35 & 00\end{array}$

2500

1500

600

400

$30 \quad 00$

$25 \quad 00$

$20 \quad 00$

2500

$20 \quad 00$

1600

1800

1500

1200 


\section{DECIDUOUS ORNAMENTAL TREES}

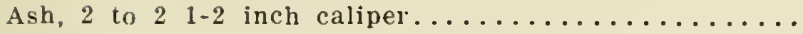

Per 100

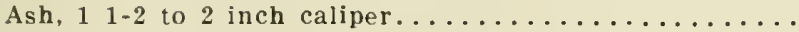

$\$ 1700$

$\$ 150 \quad 00$

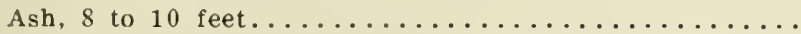

$10 \quad 00$

$90 \quad 00$

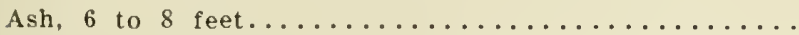

500

$45 \quad 00$

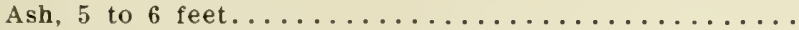

350

200

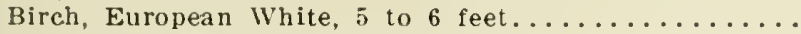

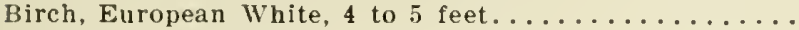

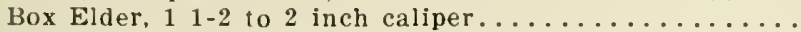

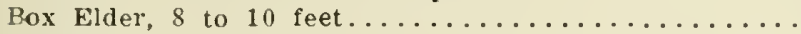

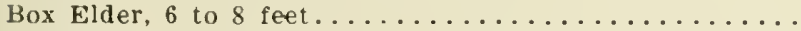

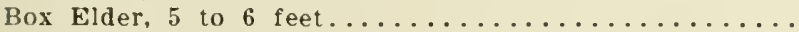

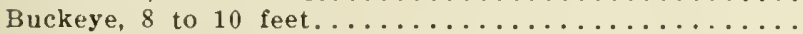

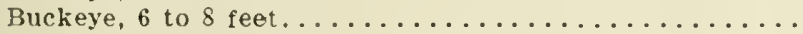

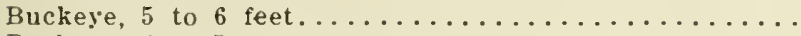

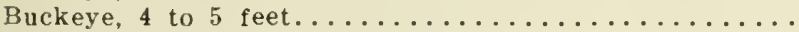

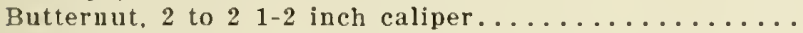

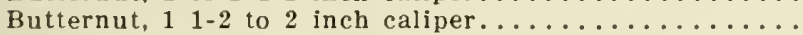

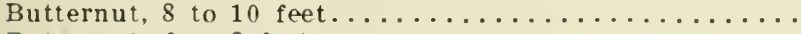

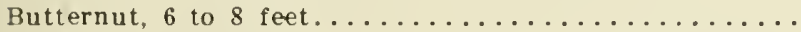

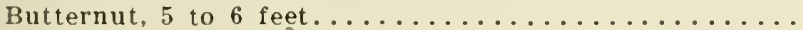

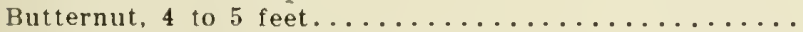

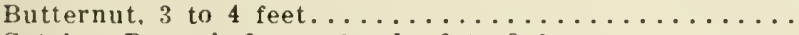

Catalpa, Bungei, 2 year heads, 6 to 8 feet..........

Catalpa, Bungei, 1 year heads, 6 to 8 feet..........

Catalpa, Bungei, 1 year heads, 5 to 6 feet...........

Catalpa, Speciosa, 1 1-2 to 2 inch caliper............

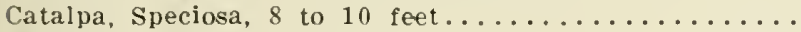

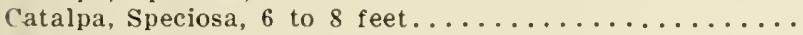

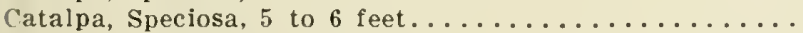

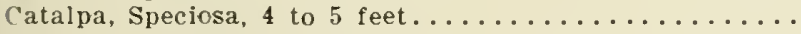

Cherry, Wild Black, 6 to 8 feet.

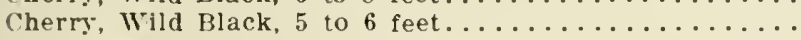

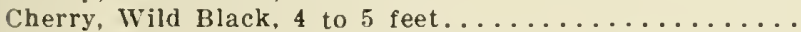

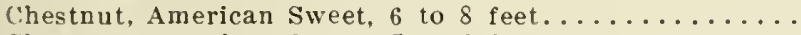

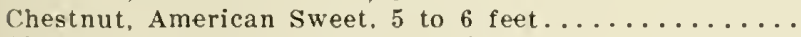

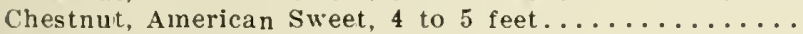

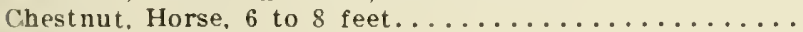

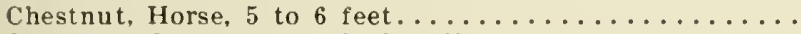

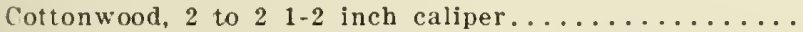

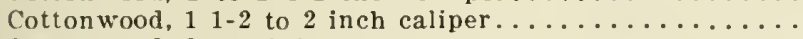

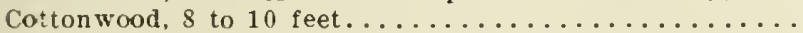

('rab, Bechtel's Double Flowering, 4 to 5 feet........

Crab, Bechtel's Double Flowering, 3 to 4 feet.........

Crab. Bechtel's Double Flowering, 2 to 3 feet.........

('rab, Bechtel's Double Flowering, 18 to 24 incles......

Elm, American White, 3 1-2 to 4 inch caliper........

Elm, American White, 3 to 3 1-2 inch caliper.........

Elm, American White, 2 1-2 to 3 inch caliper.........

Elm, American White, 2 to 2 1-2 inch caliper.........

Eln, American White, $13-4$ to 2 inch caliper.........

Elm, American White, 1 1-2 to $13-4$ inch caliper.....

Elm, American White, 1 1-4 to 1 1-2 inch caliper......

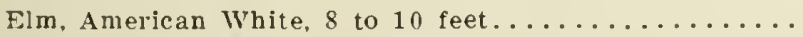

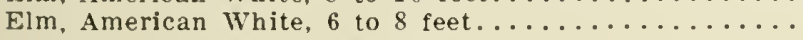

500

350

$8 \quad 50$

450

300

200

1500

$10 \quad 00$

$7 \quad 50$

500

1750

1250

$\begin{array}{ll}9 & 00\end{array}$

650

$5 \quad 00$

400

300

850

650

$5 \quad 00$

800

500

300

200

125

650

500

$4 \quad 00$

$5 \quad 50$

450

350

$10 \quad 00$

750

600

400

250

650

500

400

300

$40 \quad 00$

$30 \quad 00$

2250

1650

1300

$10 \quad 00$

$\begin{array}{ll}7 & 00\end{array}$

$30 \quad 00$

$18 \quad 00$

4500

$\begin{array}{ll}30 & 00\end{array}$

$\begin{array}{lll}75 & 00\end{array}$

$40 \quad 00$

$\begin{array}{lll}25 & 00\end{array}$

1800

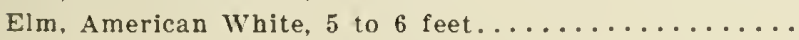

500

$\begin{array}{ll}3 & 50\end{array}$

200

$50 \quad 00$

$40 \quad 00$

$\begin{array}{ll}30 & 00\end{array}$

.....

.......

....

....

....

3500

2500

$\cdots \cdots$

$150 \quad 00$

11500

$90 \quad 00$

6500

4500

$30 \quad 00$

1800 


\section{DECIDUOUS ORNAMENTAL TREES-Continued}

Per 10

Per 100

Elm, Scotch, 3 to $31-2$ inch caliper............ \$2500

Elm, Scotch, 2 1-2 to 3 inch caliper.............. 2250

配Im, Scotch, 2 to 2 1-2 inch caliper............. 1750

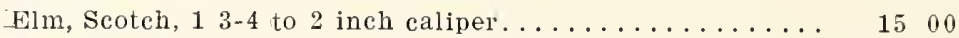

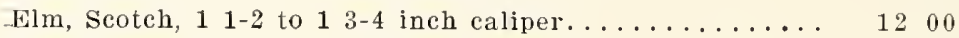

Eilm, Scotch, 1 1-4 to 1 1-2 inch caliper........... 900

Hackberry, 1 1-2 to 2 inch caliper.............. 900

Hackberry, 8 to 10 feet...................... 800

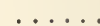

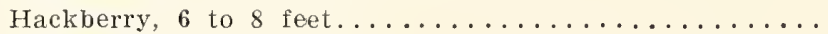

650

7500

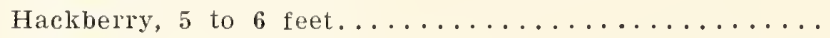

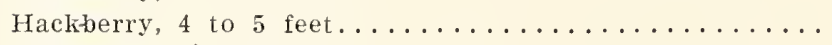

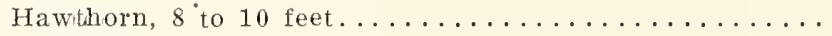

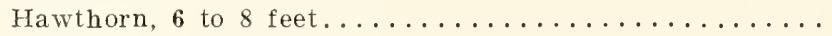

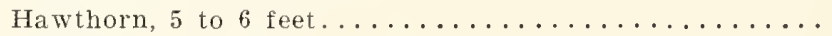

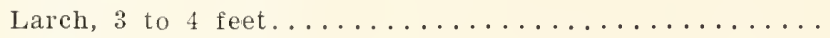

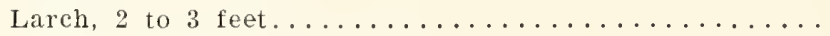

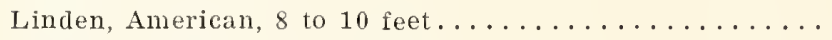

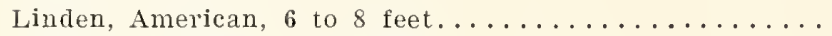

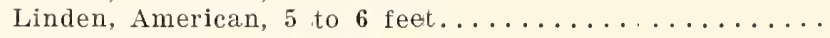

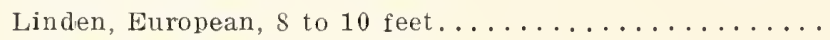

450

6000

350

$40 \quad 00$

1000

$30 \quad 00$

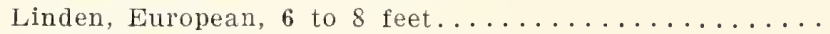

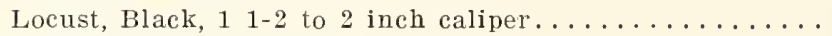

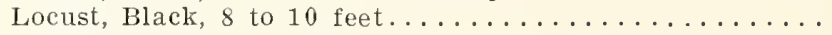

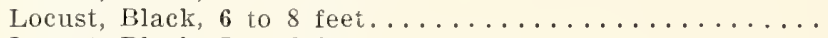

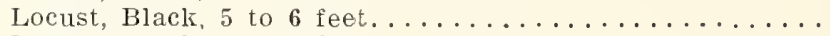

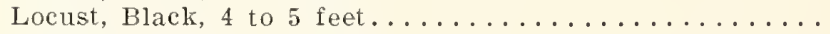

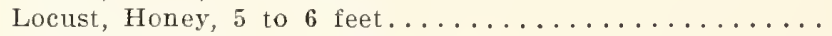

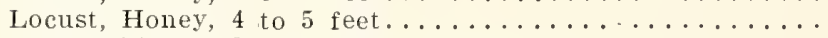

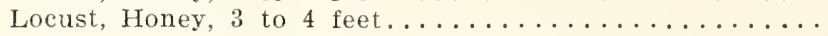

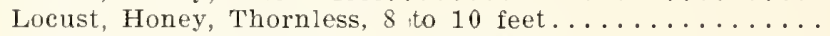

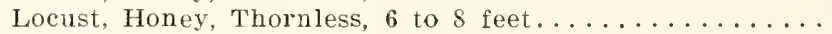

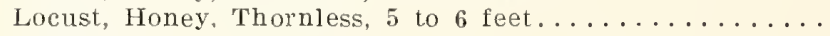

Magnolia, 8 to 10 feet.

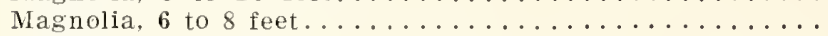

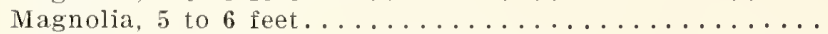

Maple, Norway, 3 to $31-2$ inch caliper............

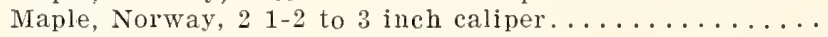

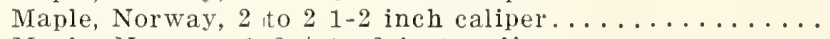

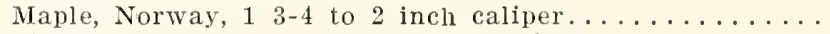

Maple, Norway, 1 1-2 to $13-4$ inch caliper...........

Maple, Norway, 1 1-4 to 1 1-2 incl caliper............

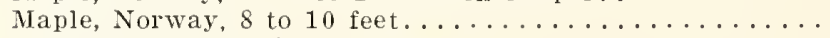

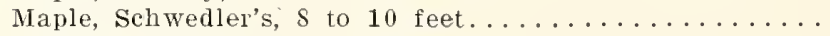

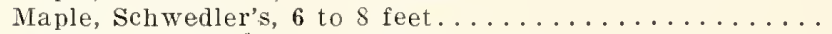

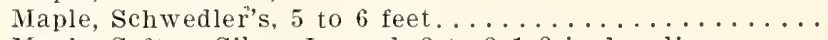

Maple, Soft or Silver Leaved, 3 to 3 1-2 inch caliper......

Maple, Soft or Silver Leaved, 2 1-2 to 3 inch caliper.....

Maple, Sofit or Silver Leaved, 2 to 2 1-2 inch caliper......

Maple, Soft or Silver Leaved, 1 1-2 to 2 inch caliper......

Maple, Soft or Silver Leaved, 8 to 10 feet..........

Maple, Soft or Silver Leaved, 6 to $s$ feet............

Maple, Soft or Silver Leaved, 5 to 6 feet............

750

650

450

350

900

750

500

900

750

650

450

300

175

125

350

250

175

650

500

350

1000

S 50

700

$\begin{array}{ll}35 & 00\end{array}$

$\begin{array}{lll}30 & 00\end{array}$

2250

1750

1450

1200

$10 \quad 00$

1750

1500

1250

$25 \quad 00$

1750

1500

750

450

300

175 


\section{DECIDUOUS ORNAMENTAL TREES-Continued}

Maple, Sugar or Hard, 2 to 2 1-2 inch caliper........... Maple. Sugar or Hard, $13-4$ to 2 inch caliper.......... Maple, Sugar or Hard, 1 1-2 to $13-4$ inch caliper....... Maple, Sugar or Hard, 1 1-4 to 1 1-2 inch caliper...... Maple, Sugar or Hard, 8 to 10 feet..............

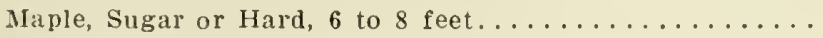

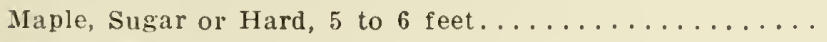
Maple, Weir's Cut Leaved, $11-2$ to 2 inch caliper....... Maple, Weir's Cut Leaved, 1 1-4 to 1 1-2 inch caliper..... Maple, Weir's Cut Leaved, 8 to 10 feet. . . . . . . . . . . . Maple, Weir's Cut Leaved, 6 to 8 feet.............. Maple, Weir's Cut Leaved, 5 to 6 feet............... Maple, Weir's Cut Leaved, 4 to 5 feet............... Mountain Ash, European, 2 to 2 1-2 inch caliper........ Mountain Ash, European, 1-2 to 2 inch caliper........ Mountain Ash, European, 8 to 10 feet............ Mountain Ash, European, 6 to 8 feet.

Mountain Ash, European, 5 to 6 feet..............

Mountain Ash, European, 4 to 5 feet.............

Mountain Ash, Oak Leaved, 6 to 8 feet..............

Mountain Ash, Oak Leaved, 5 to 6 feet..............

Mountain Ash, Oak Leaved, 4 to 5 feet..............

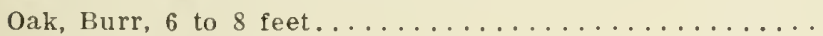

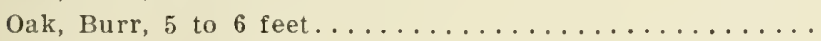

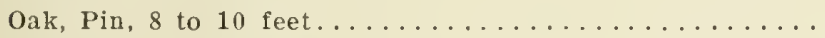

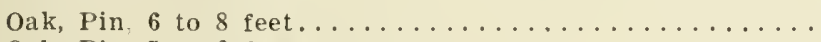

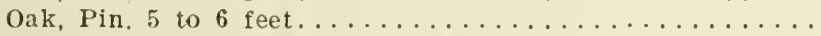

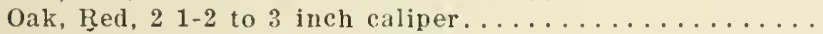

Oak, Red, 2 to 2 1-2 inch caliper................

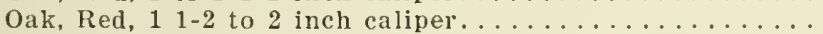

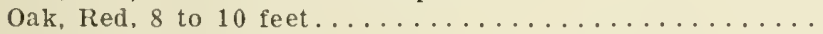

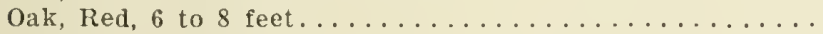

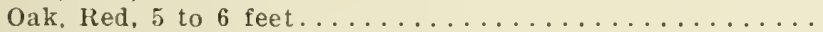

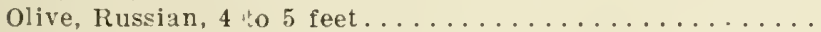

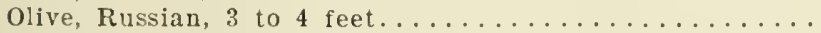

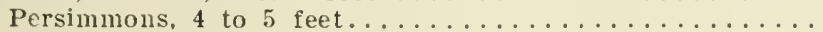

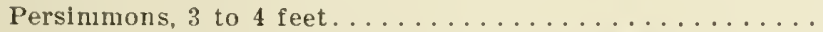

Poplar, Balm of Gilead, 2 to $21-2$ inch caliper.........

Poplar, Balm of Gilead, 1 1-2 to 2 inch caliper.........

Poplar, Balm of Gilead, 1 1-4 to 1 1-2 inch caliper......

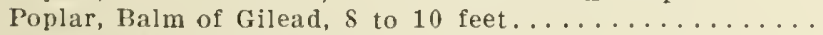

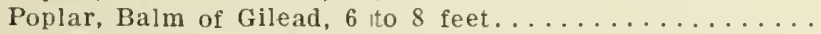

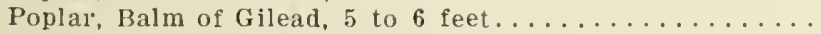

Poplar, Balm of Gilead, 4 to 5 feet...............

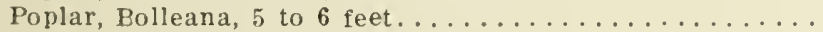

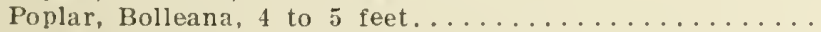

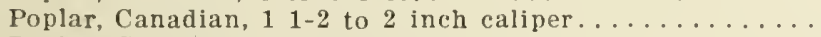

Poplar, Canadian, 1 1-4 to 1 1-2 inch caliper...........

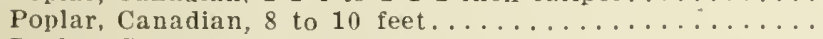

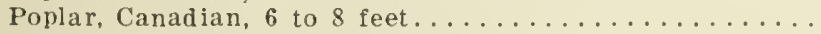

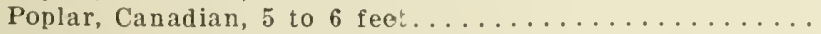

Per 10

$\$ 1750$

1600

1350

1100

900

700

450

1250

$10 \quad 00$

850

$5 \quad 50$

400

250

1250

$10 \quad 00$

850

550

400

300

750

600

400

850

700

1400

1000

750

$30 \quad 00$

2500

1500

1250

$10 \quad 00$

$7 \quad 50$

350

250

500

350

$10 \quad 00$

750

400

300

200

140

125

400

300

850

450

350

225

150
Per 100

$150 \quad 00$

12500

$100 \quad 00$

8500

6500

$40 \quad 00$

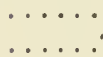

7500

$50 \quad 00$

3500

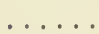

$75 \quad 00$

$50 \quad 00$

3500

2500
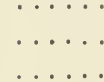

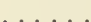
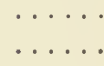

$\cdots \ldots$

$\ldots \ldots$

....

$\ldots \ldots$

$\ldots \ldots$

$\cdots \ldots$

$\ldots \ldots$

$\ldots \ldots$

6500

3500

2500

1800

1250

1000

\section{......}

7500

$40 \quad 00$

$30 \quad 00$

$20 \quad 00$

1250 


\section{DECIDUOUS ORNAMENTAL TREES-Continued}

Poplar, Carolina, 3 to 3 1-2 inch caliper.......... $\$ 1500$

Poplar, Carolina, 2 1-2 to 3 inch caliper........... 1250

Poplar, Carolina, 2 to 2 1-2 inch caliper........... 1000

Poplar, Carolina, 1 1-2 to 2 inch caliper............ 600

$90 \quad 00$

Poplar, Carolina, 1 1-4 to 1 1-2 inch caliper..........

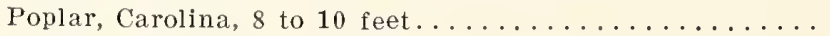

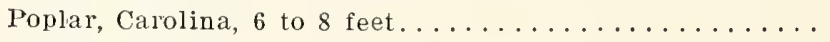

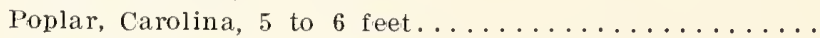

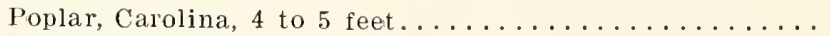

$\begin{array}{llll}3 & 50 & 30 & 00\end{array}$

$225 \quad 20 \quad 00$

$170 \quad 15 \quad 00$

$90 \quad 8 \quad 00$

$\begin{array}{lll}70 & 6 & 00\end{array}$

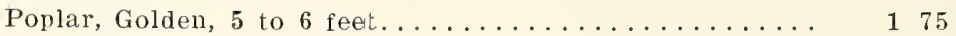

1500

Poplar, Golden, 4 to 5 feet.................. 140

Poplar, Lombardy, 3 to 3 1-2 inch caliper.......... 1500

Poplar, Lombardy, $21-2$ to 3 inch caliper........... 1250

Poplar, Lombardy, 2 to $21-2$ inch ealiper................... 1000

Poplar, Lombardy, 1 1-2 to 2 inch caliper...........

Poplar, Lombardy, 1 1-4 to 1 1-2 inch caliper.........

Poplar, Lombardy, 8 to 10 feet...............

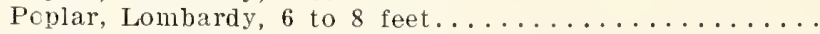

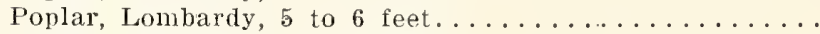

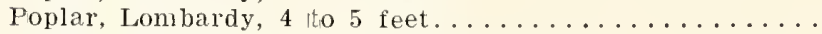

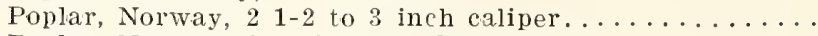

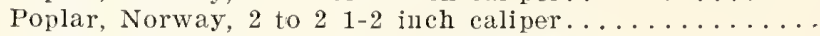

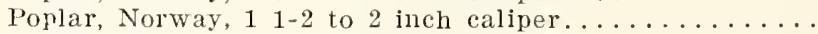

Poplar, Norway, 1 1-4 to 1 1-2 inch caliper..........

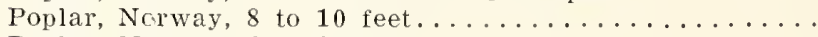

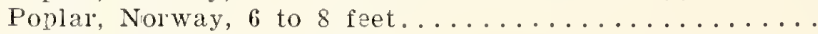

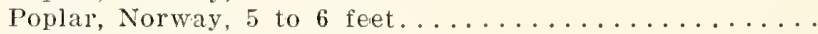

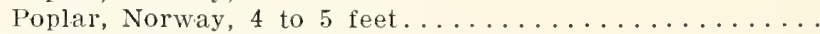

Poplar, Silver-Leaved, 1 1-2 to 2 inch caliper..........

Poplar, Silver-Leaved, 8 to 10 feet..............

Poplar, Silver-Leaved, 6 to 8 feet..............

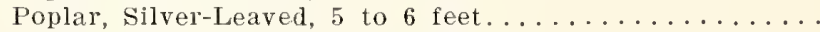

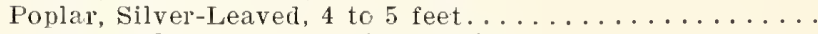

Poplar, Volga, 2 1-2 to 3 inch caliper.............

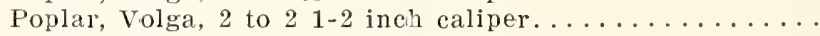

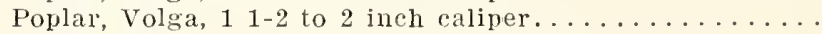

Poplar, Volga, 1 1-4 to 1 1-2 inch caliper...........

Poplar, Volga, 8 to 10 feet..................

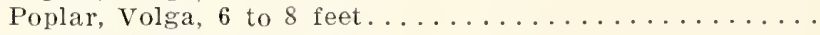

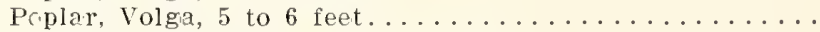

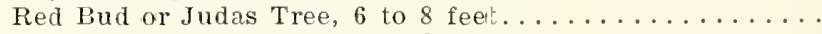

Red Bud or Judas Tree, 5 to 6 fest...............

Red Bud or Judas Tree, 4 to 5 feet..............

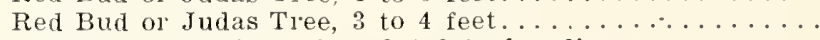

Sycamore, American, 2 to 2 1-2 inch caliper.........

Sycamore, American. 1 1-2 to 2 inch caliper..........

Sycamore, American, 1 1-4 to 1 1-2 inch caliper........

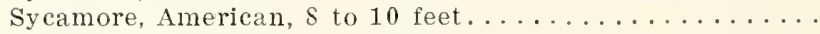

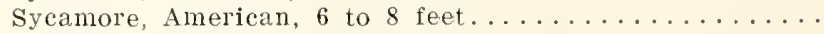

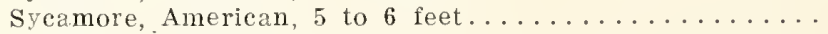

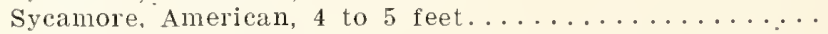

Tulip Tree, 8 to 10 feet. . . . . . . . . . . . .

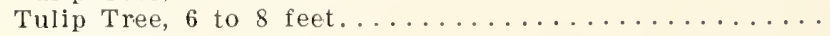

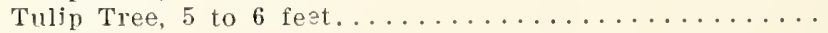

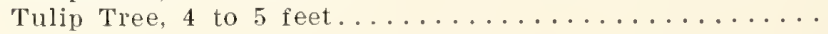

$6 \quad 50$

400

300

200

150

90

1250

$10 \quad 00$

$6 \quad 00$

350

250

175

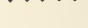

....

$\begin{array}{lll}60 & 00\end{array}$

3500

2500

1800

1250

800

9000

$\begin{array}{ll}50 & 00\end{array}$

3000

$\begin{array}{ll}20 & 00\end{array}$

$\begin{array}{ll}15 & 00\end{array}$

800

600

300

850

$6 \quad 50$

$5 \quad 00$

$4 \quad 00$

.....

$\ldots \ldots$

2000

1500

$\ldots$

$60 \quad 00$

3500

2500

1s 00

1200

......

....

$\ldots \ldots$

$\ldots \ldots$

$\ldots \ldots$

5500

$\begin{array}{lll}40 & 00\end{array}$

30 ก ก

2500

$\ldots .$.

$\ldots \ldots$ 


\section{DECIDUOUS ORNAMENTAL TREES-Continued}

Per 10

\section{WEEPING TREES}

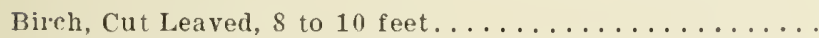

Birch, Cut Leaved, 6 to 8 feet...............

Birch, Cut Leaved, 5 to 6 feet...............

Elm, Camperdown, 2 year heads..............

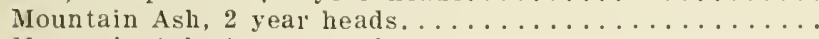

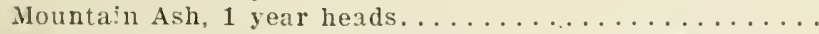

Mulberry, Tea's Weeping Russian, 2 year heads.......

Mulberry, Tea's Weeping Russian, 1 year heads.......

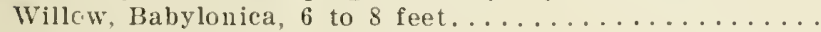

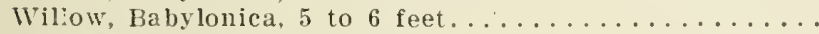

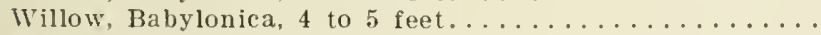

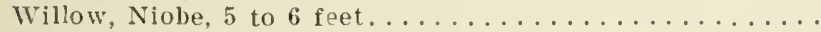

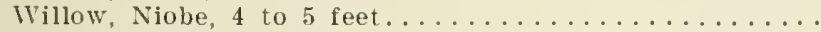

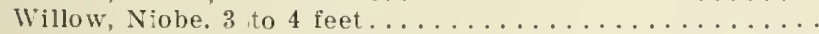

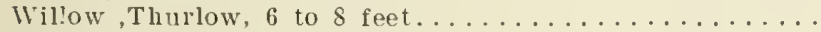

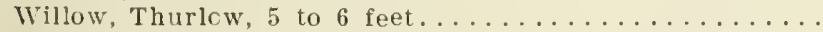

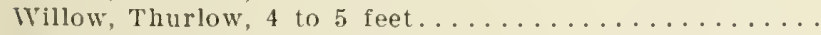

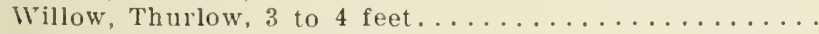

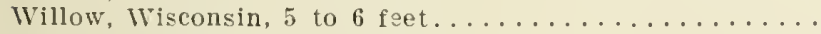

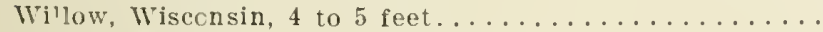

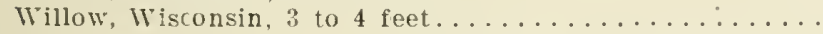

\section{ORNAMENTAL SHRUBS}

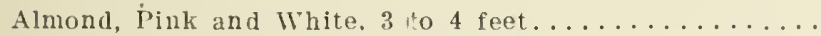

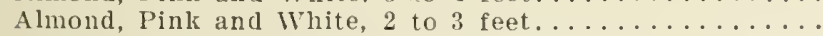

Almond, Pink and White, 18 to 24 inches...........

Altheas, Bush, Assorted Varieties, 3 to 4 feet.........

Altheas, Bush, Assorted Varieties, 2 to 3 feet.........

Altheas, Bush, Assorted Varieties, 18 to 24 inches........

Altheas, Tree, Assorted Varieties, 4 to 5 feet..........

Altheas, Tree, Assorted Varieies, 3 to 4 feet..........

Aralia Pentaphylla, 3 to 4 feet.................

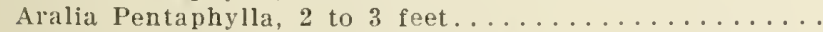

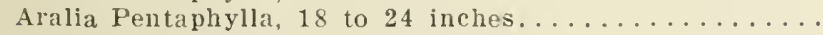

Palber'ry Thumberg's, 2 1-2 to 3 feet...............

Barberry Thunberg's, 2 to 2 1-2 feet..............

Barberry Thunberg's, 18 to 24 inches.
$\$ 1250$

900

750

$5 \quad 50$

400

300

600

200

150

90

60

200

125

75

Per 100

$\begin{array}{rr}\cdots & \cdots \\ 70 & 00 \\ 50 & 00 \\ 35 & 00 \\ 25 & 00 \\ \cdots \cdots \\ \cdots & \cdots \\ 12 & 50 \\ 8 & 00 \\ 5 & 00 \\ 18 & 00 \\ 10 & 00 \\ 6 & 00\end{array}$

Per 10

Per 100

$\$ 1250$

$10 \quad 00$

750

1500

1250

$10 \quad 00$

$15 \quad 00$

1250

300

250

200

200

150

125

300

250

150

100

225

150

100

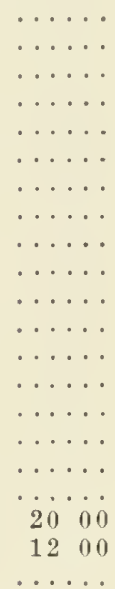

Per 10

Per 100

$\$ 475$

375

325

240

180

140

450

325

275

225

175

325

225

180

3500

$\begin{array}{ll}30 & 00\end{array}$

2200

1600

1200

$40 \quad 00$

$30 \quad 00$

2500

$20 \quad 00$

1500

$30 \quad 00$

$\begin{array}{ll}20 & 00\end{array}$

1600 


\section{ORNAMENTAL SHRUBS-Continued}

Buckthorn, 2 to 3 feet.

Per 10

$\$ 100$

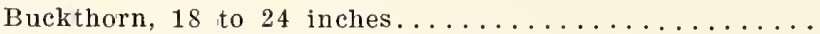

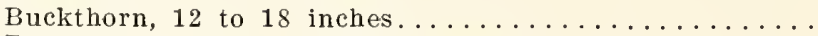

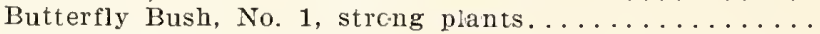

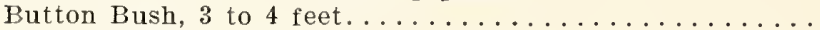

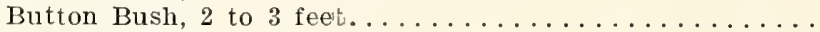

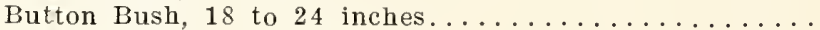

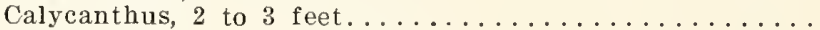

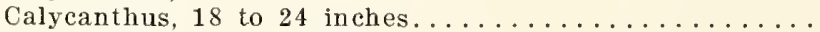

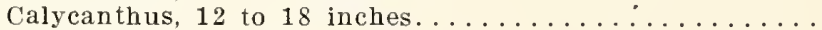

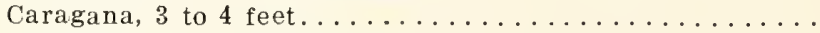

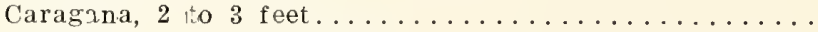

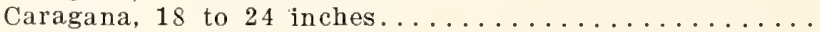

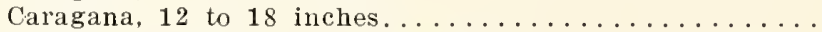

Clethra, Alnifolia, 18 to 24 inches.............

Clethra, Alnifolia, 12 to 18 inches.............

Cornus, Elegantissima, variegated, 3 to 4 feet.........

Cornus, Elegantissima, variegated, 2 to 3 feet.........

Cornus, Elegantissima, variegated, 18 to 24 inches......

Cornus, Florida, 3 to 4 feet.................

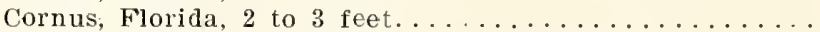

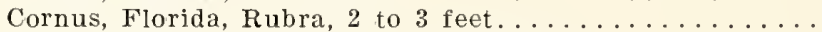

Cornus, Florida, Rubra, 18 to 24 inches............

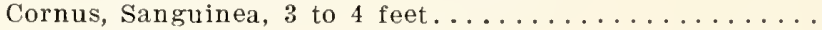

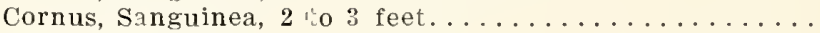

Cornus, Sanguinea, 18 to 24 inches..............

Cornus, Sanguinea, 12 to 18 inches.............

Cornus, Siberica, 3 to 4 feet.................

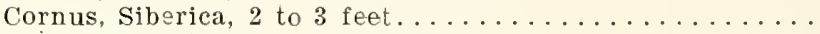

Cornus, Siberica, 18 to 24 inches..............

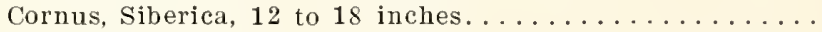

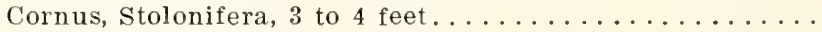

Cornus, Stolonifera, 2 to 3 feet...............

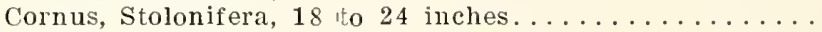

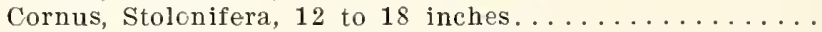

Cornus, Stolonifera, Lutea, 18 to 24 inches...........

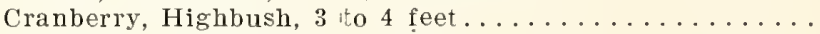

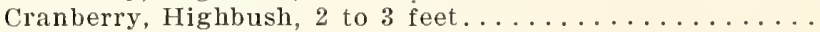

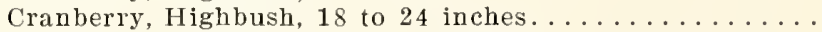

Cranberry, Highbush, 12 to 18 inches.............

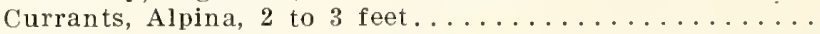

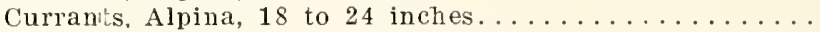

Currants, Yellow Flowering, 3 to 4 feet............

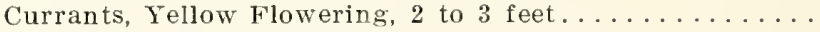

Currants, Yellow Flowering, 18 to 24 inches..........

Desmodium, Penduliflorum, ..............

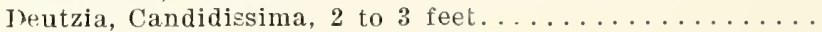

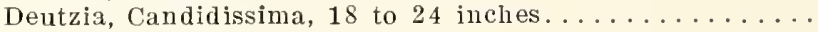

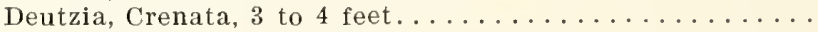

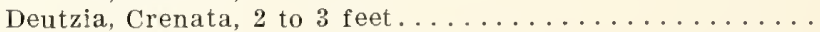

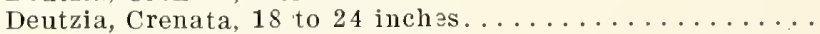

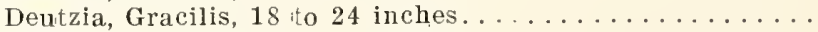

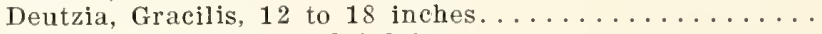

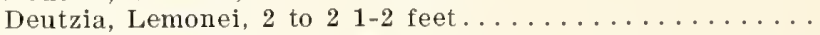

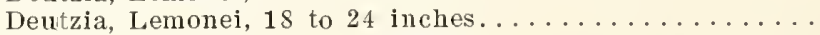

Deutzia, Lemonei, 12 to 18 inches.
Per 100

$\$ 850$

750

$5 \quad 00$

2000

225

250

200

150

2 75

180

150

$2 \quad 25$

175

125

80

200

150

350

300

250

300

250

500

400

$2 \quad 25$

180

140

125

240

200

150

120

$2 \quad 25$

1 So

140

110

200

250

$2 \quad 25$

175

125

300

200

300

250

200

250

200

150

250

200

150

275

$2 \quad 25$

300

250

200
$25 \quad 00$

$20 \quad 00$

1500

$10 \quad 00$

700

.....

.

$\ldots \ldots$

$\ldots$.

.....

$\ldots \ldots$

$\ldots \ldots$

$\ldots .$.

.

$\ldots \ldots$

2200

1800

1400

$\begin{array}{lll}20 & 00\end{array}$

1600

1200

$10 \quad 00$

$20 \quad 00$

1500

$10 \quad 00$

......

$\ldots \ldots$

$\ldots \ldots$

$\ldots \ldots$

is 00

1250

2200

$\begin{array}{ll}18 & 00\end{array}$

1250

2500 


\section{ORNAMENTAL SHRUBS-Continued}

Deutzia, Pride of Rochester, 3 to 4 feet............

Deutzia, Pride of Rochester, 2 to 3 feet.............

Deutzia, Pride of Rochester, 18 to 24 inches..........

Deutzia, Pride of Rochester, 12 to 18 inches..........

Deutzia, Rosea, 18 to 24 inches................

Deutzia, Rosea, 12 to 18 inches................

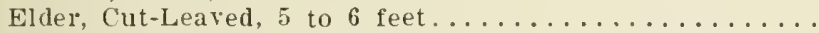

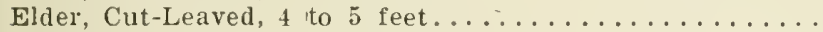

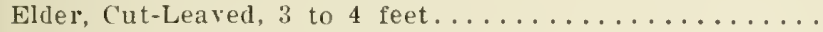

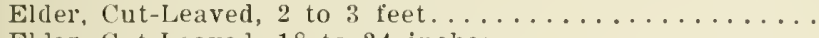

Elder, Cut-Leaved, 18 to 24 inches..............

Elder, Golden-Leaved, 4 to 5 feet..............

Elder, Golden-Leaved, 3 to 4 feet...............

Elder, Golden-Leaved, 2 to 3 feet...............

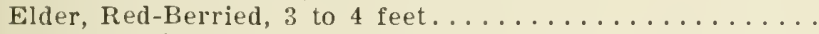

Elder, Red-Berried, 2 to 3 feet...............

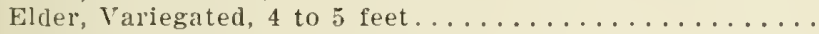

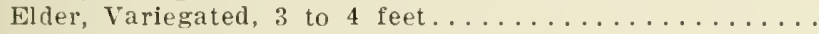

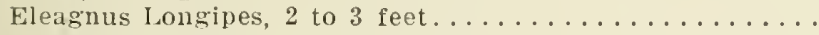

Eleagnus Longipes, 18 to 24 inches..............

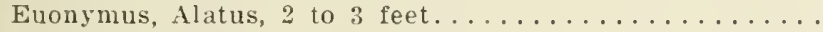

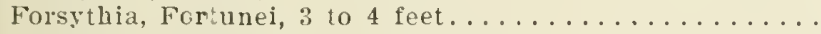

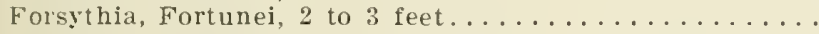

Forsythia, Fortumei, 18 to 24 inches.............

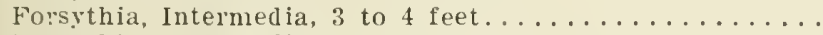

Forsythia, Intermedia, 2 to 3 feet..............

Forsythia, Intermedia, 18 to 24 inches............

Forsythia, Intermedia, 12 to 18 inches...........

Forsythia, Suspensa, 3 to 4 feet...............

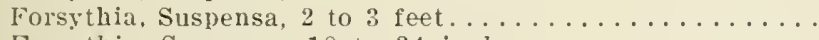

Frrsythia, Suspensa, 18 to 24 inches............

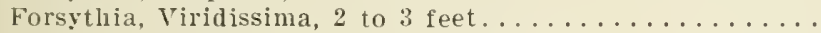

Forsythia, Viridissima, 18 to 24 inches............

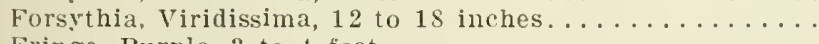

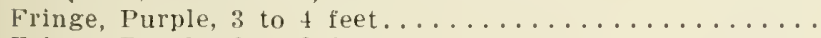

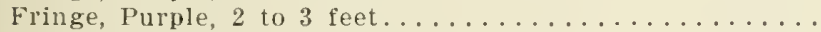

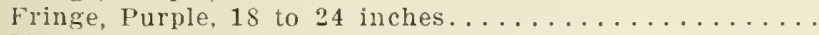

Honeysuckle, Bella Albida, 2 to 3 feet.............

Honeysuckle, Bella Albida, 18 to 24 inches...........

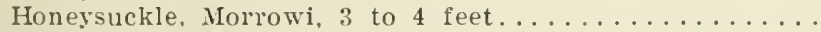

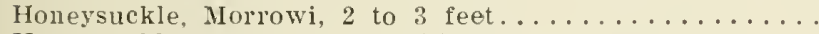

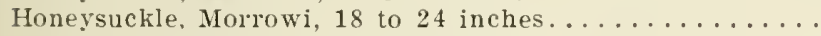

Honeysuckle, Morrowi, 12 to 18 inches..............

Honeysuckle, Tartarian, Pink and Red, 3 to 4 feet.......

Honeysuckle, Tartarian, Pink and Red, 2 to 3 feet......

Honeysuckle, Tartarian. Pink and Red, 18 to 24 inches....

Honeysuckle, Tartarian, White, 3 to 4 feet..........

Honeysuckle, Tartarian, White, 2 to 3 feet..........

Honeysuckle. Tartarian, White, 18 to 24 inches........

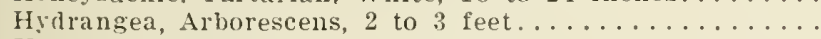

Hydrangea, Arborescens, 18 to 24 inches...........

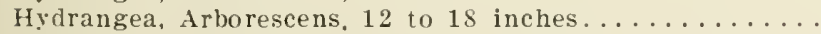

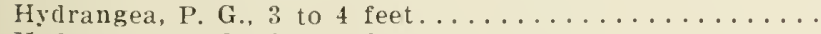

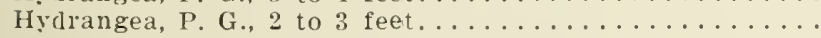

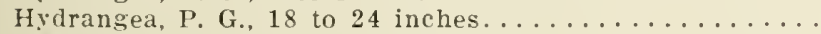

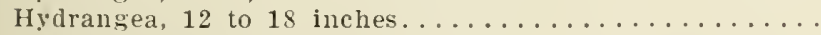

Hydrangea, Tree Form, 4 to 5 feet.............

Hydrangea, Tree Form. 3 to 4 feet.

Per 10

225

180

140

90

140

90

350

300

250

200

175

325

275

225

250

200

300

250

250

200

350

240

200

160

225

180

160

135

250

225

170

180

140

90

325

275

200

200

160

240

200

160

125

240

200

160

240

200

160

375

275

200

425

375

275

200

750

600
Per 100

$\begin{array}{lll}20 & 00\end{array}$

1600

1200

800
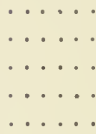

$\begin{array}{ll}30 & 00\end{array}$

2500

$20 \quad 00$

....

$\ldots$

$\ldots \ldots$

$\ldots \ldots$

2200

1800

1400

2000

1600

$1+0$

1200

$20 \quad 0$ 日

1500

1600

1200

800

$30 \quad 00$

2500

1800

$1 \mathrm{~s} 00$

1400

2200

1800

140 ด

1000

2200

1800

1400

2200

1800

1400

3500

2500

1800

$40 \quad 00$

3500

2500

$1 \mathrm{~S} 90$ 


\section{ORNAMENTAL SHRUBS-Continued}

Kerria, Japonica, 3 to 4 feet................

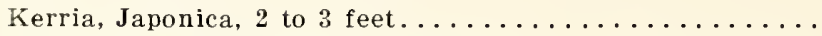

Lilacs, Named Varieties, 4 to 5 feet.

$3 \quad 50$

300

Lilacs, Named Varieties, 3 to 4 feet..............

500

$4 \quad 00$

Lilacs, Named Varieties, 2 to 3 feet..............

$\begin{array}{lll}3 & 00\end{array}$

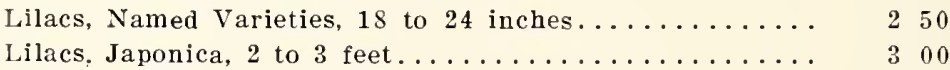

Lilacs, Josikaea, 3 to 4 feet................. 400

Lilacs, Josikaea, 2 to 3 feet................. 300

Lilacs, Persian Purple, 3 to 4 feet.............. '3 25

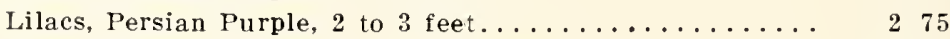

Lilacs, Persian Purple, 18 to 24 inches............ 225

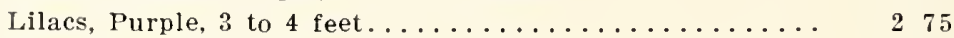

Lilacs, Purple, 2 to 3 feet................... 225

Lilacs, Purple, 18 to 24 inches................ 175

Lilacs, Purple, 12 to 18 inches................ 125

Lilacs, Rothomagensis, 3 to 4 feet............... 325

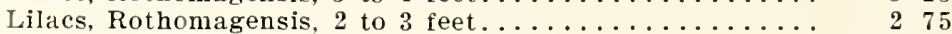

Lilacs, Rothomagensis, 18 to 24 inches.............

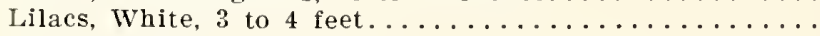

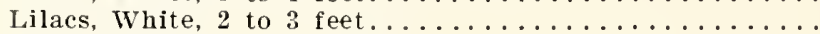

Lilacs, White, 18 to 24 inches...............

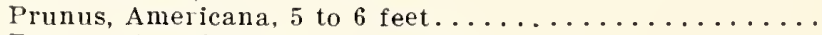

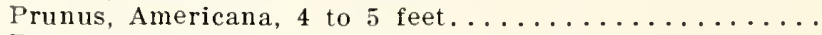

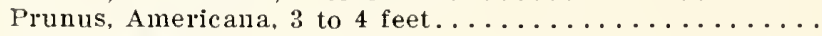

Prunus, Othello, 5 to 6 feet................

Prunus, Othello, 4 to 5 feet.................

Prunus, Othello, 3 to 4 feet.................

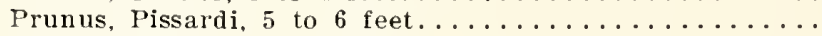

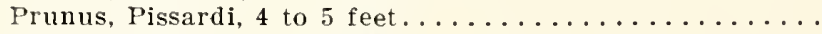

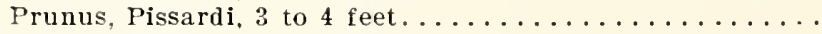

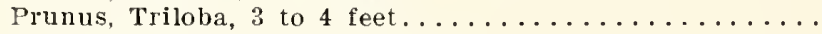

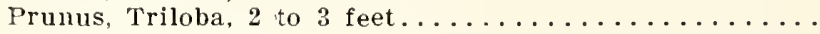

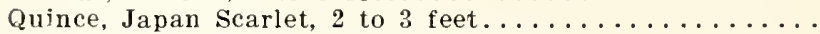

Quince, Japan Scarlet, 18 to 24 inches.............

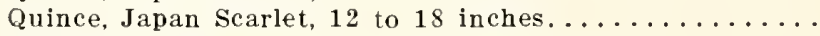

Rhododendron

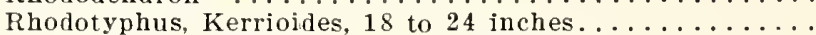

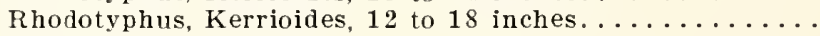

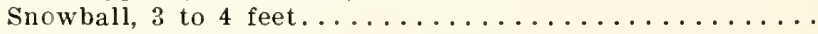

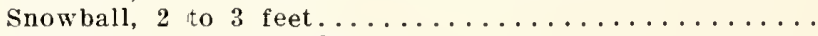

Snowball, 18 to 24 inches..................

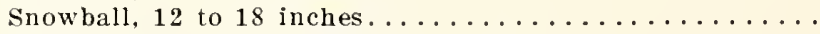

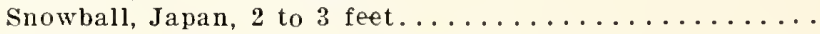

Snowball, Japan, 18 to 24 inches..............

Snowberry, Red, Indian Currant, 3 to 4 feet.........

Snowberry, Red, Indian Currant, 2 to 3 feet..........

Snowberry, Red, Indian Currant, 18 to 24 inches........

Snowberry, Red, Indian Currant, 12 to 18 inches.......

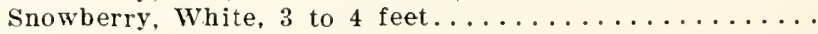

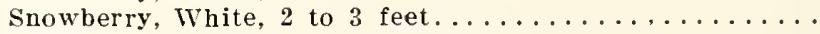

Snowberry, White, 18 to 24 inches..............

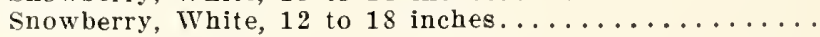




\section{ORNAMENTAL SHRUBS-Continued}

Gpirea, Anthony Waterer, 2 to 2 1-2 feet............

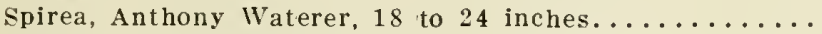

Spirea, Anthony Waterer, 12 to 18 inches...........

Spirea, Arguta, 18 to 24 inches...............

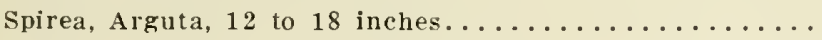

Spirea, Billardi, Pink, 3 to 4 feet...............

Spirea, Billardi, Pink, 18 to 24 inches............

Spirea, Billardi, Pink, 12 to 18 inches............

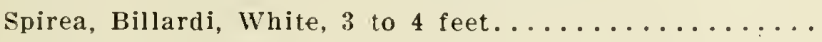

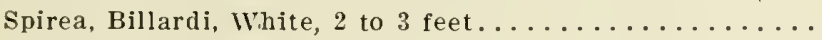

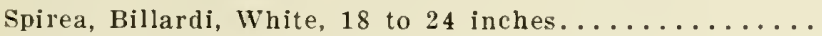

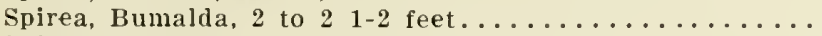

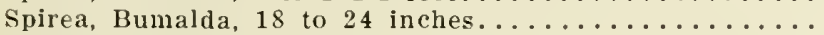

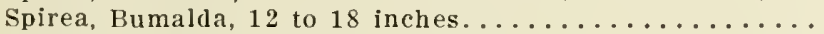

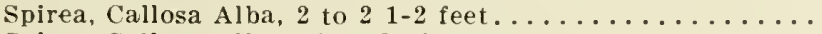

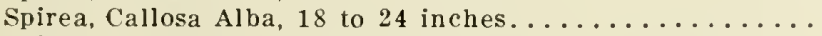

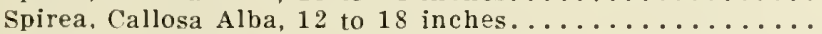

Spirea, Callosa Rosea, 3 to 4 feet................

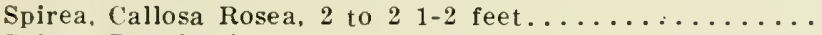

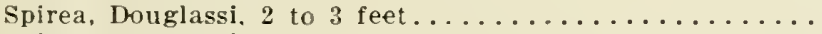

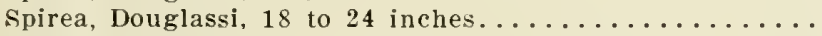

Spirea, Opulifolia, 3 to 4 feet...............

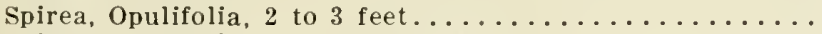

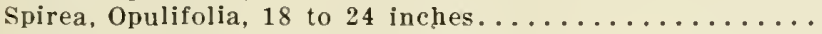

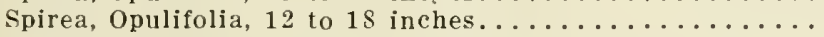

Spirea, Opulifolia, Aurea, 3 to 4 fee $\ldots \ldots \ldots \ldots \ldots \ldots$

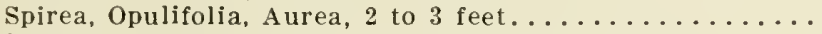

Spirea, Opulifolia, Aurea, 18 to 24 inches...........

Spirea, Opulifclia, Aurea, 12 to 18 inches............

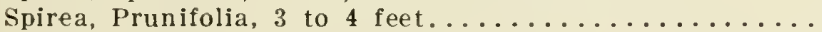

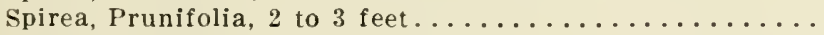

Spirea, Prunifolia, 18 to 24 inches..............

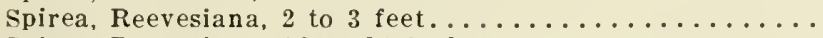

Spirea, Reevesiana, 18 to 24 inches..............

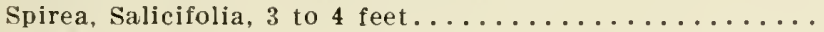

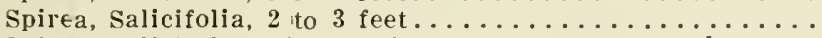

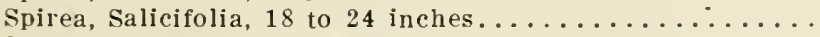

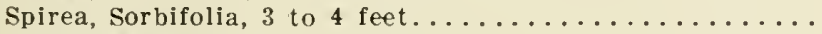

200

1750

160

1400

375

275

225

325

240

1 S 0

375

325

175

140

225

170

3500

2500

$20 \quad 00$

$30 \quad 00$

2200

1600

$30 \quad 00$

1500

1200

$20 \quad 00$

1500

140

110

$2 \quad 25$

170

1200

900

$20 \quad 00$

1500

140

110

325

275

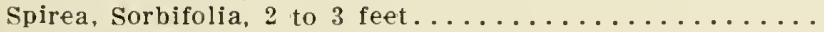

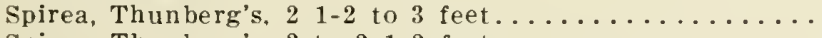

Spirea, Thunberg's, 2 to 2 1-2 feet..............

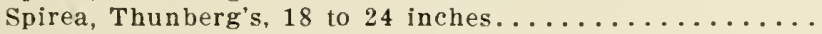

Spirea, Thunberg's, 12 to 18 inches..............

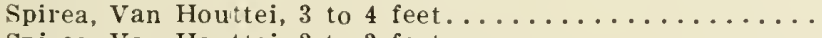

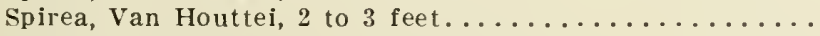

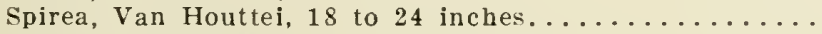

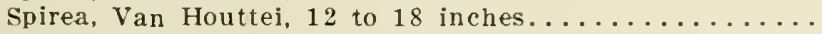

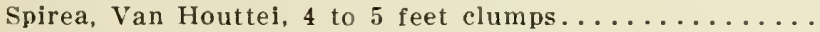

Spirea, Van Houttei, 3 to 4 feet clumps.............

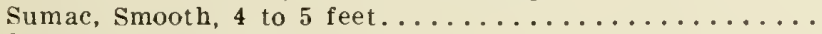

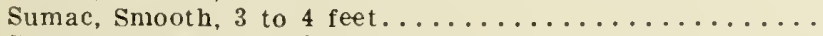

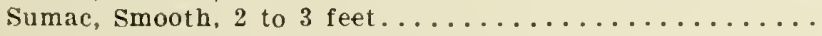




\section{ORNAMENTAL SHRUBS-Continued}

Sumac, Staghorn, 3 to 4 feet.

Sumac, Staghorn, 2 to 3 feet.................

Sumac, Staghorn, Cut Leaved, 3 to 4 feet. . . . . . . . . . . .

Sumac, Staghorn, Cut Leaved, 18 to 24 inches..........

Syringa, Coronarius, 3 to 4 feet...............

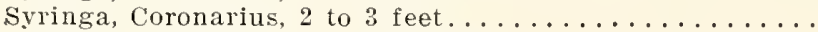

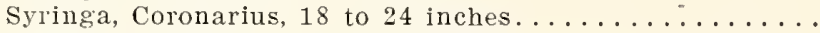

Syringa, Coronarius, 12 to 18 inches.............

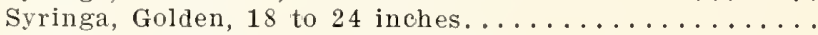

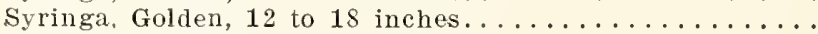

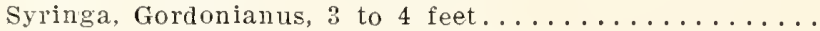

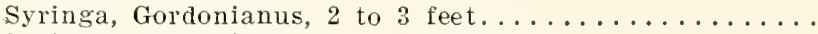

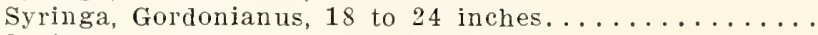

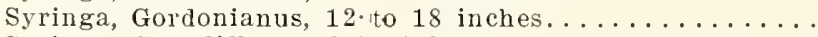

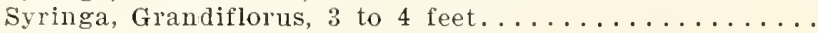

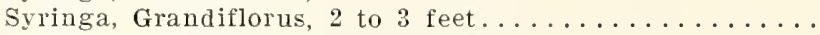

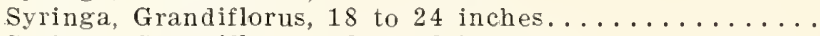

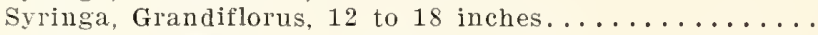

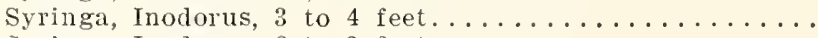

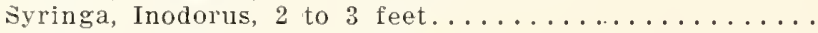

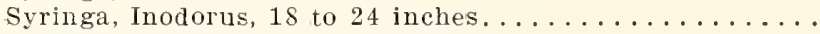

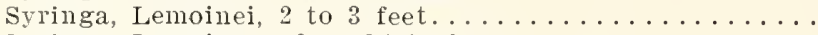

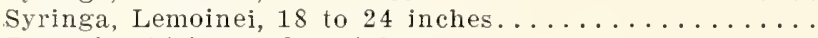

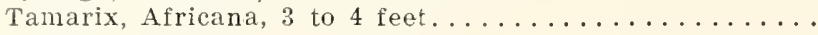

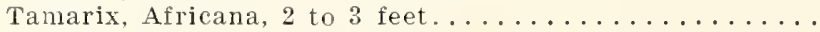

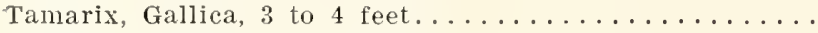

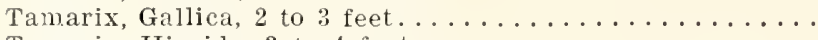

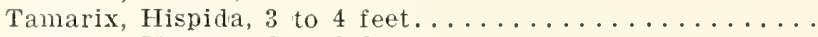

Tamarix, Hispida, 2 to 3 feet...............

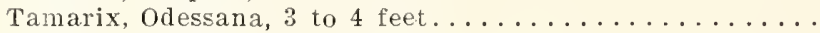

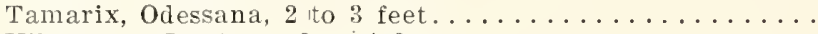

Viburnum, Lantana, 3 to 4 feet................

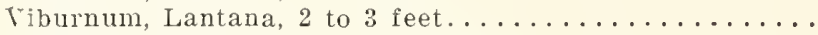

Viburnum, Lantana, 18 to 24 inches..............

Tiburnum, Lantana, 12 to 18 inches...............

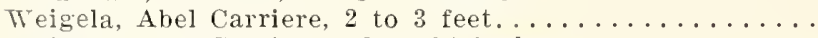

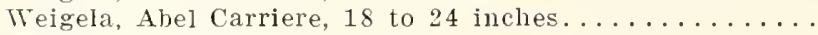

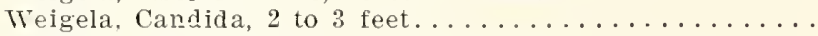

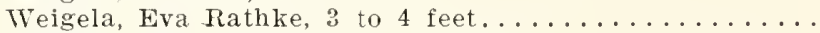

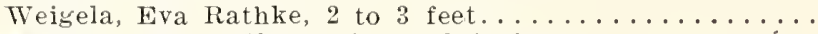

Weigela, Eva Rathke, 12 to 18 inches.............

Weigela, Eva Rathke, 12 to 18 inches.............

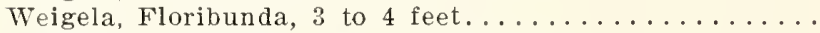

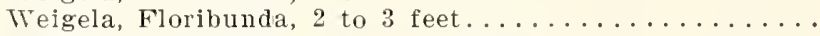

Weigela, Floribunda, 18 to 24 inches.............

Wreigela, Floribunda, 12 to 18 inches.............

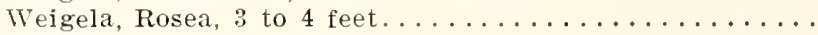

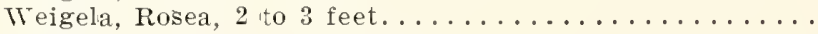

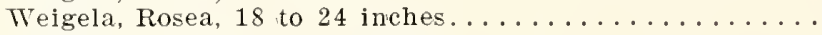

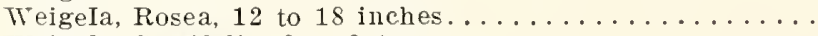

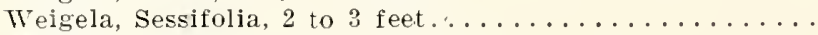

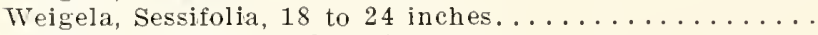

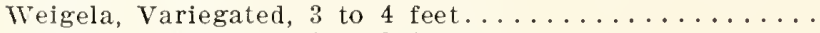

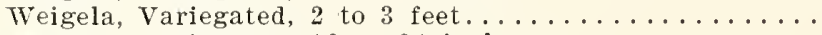

Weigela, Variegated, 18 to 24 inches.............

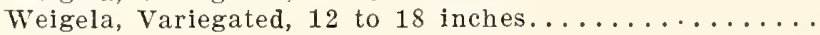

140

240

200

170

140

300

200

225

170

140

100

225

170

140

100

$2 \quad 25$

170

140

275

$2 \quad 25$

225

170

$2 \quad 25$

170

250

200

225

170

300 


\section{HEDGING}

(Lighter stock than regular grade of shrubs)

Caragana, 18 to 24 inches

Per 1000

$\$ 40 \quad 00$

3500

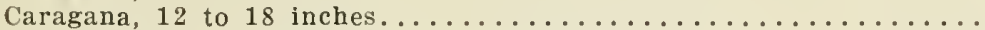

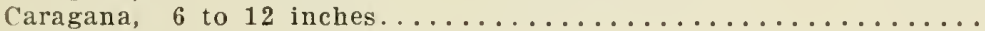

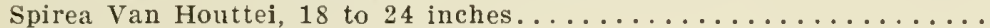

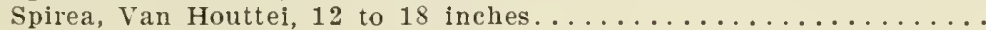

$55 \quad 00$

\section{PRIVET}

Amoor, River North, 3 to 4 feet, 4 canes and up........... Per 100

Per 100 Per 1000

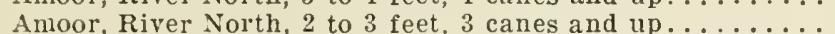

1400

Amoor, River North, 2 to 3 feet, 2 canes.............

Amoor, River North, 18 to 24 inches, 2 canes and up.....

Amoor, River North, 12 to 18 inches, 1 cane and up......

California, 3 to 4 feet, 4 canes and up.............

1200

1200

$8 \quad 00$

$6 \quad 00$

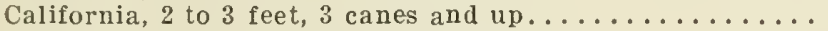

Califoruia, 18 to 24 inches, 2 canes and up...........

$5 \quad 50$

450

California, 12 to 18 inches, 1 cane and up...........

$3 \quad 25$

$1 \ddot{2} \dot{5} \ddot{0} 0$

10000

$100 \quad 00$

$75 \quad 00$

$50 \quad 00$

$40 \quad 00$

$30 \quad 00$

\section{ROSES-Hybrid Perpetual}

Assorted as follows, No. 1

\section{Alfred Colomb}

Alna de Diesbach

Barouess Rotischild

Baron de Boustettin

Captain Christy

Captain Ilayward

Clio

Coquette des Alps

Assorted as above No. $11-2$

\section{Earl of Dufferin}

Frau Karl Druschki

General Jacqueminot

Gloire de Margottin

Iugh Dickson

J. B. Clark

iad. Gabriel Luizet

Iadam Charles fiood

\section{Per 10}

$\$ 450$

Magna Charta

Margaret Diclison

Marshall $\mathrm{P}$. Wrilder

Mrs. John Laing

Panl Neyron

$P$. C. de Kohan

Uirich Brunner

\section{MISCELLANEOUS-No. 1}

(Except as noted)

$$
\$ 350
$$
)

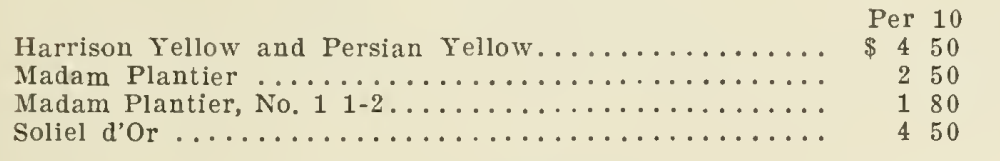

Per 100

$\$ 40 \quad 00$

$\ldots \ldots$

$\ldots \ldots$

\section{MOSS}

Assorted as follows, No. $1 \ldots \ldots \ldots \ldots \ldots \ldots \ldots \ldots$

Per 10 Per 100

$\$ 500 \quad \$ 4500$

Crested Moss

Henry Martin

Salet

\section{RUGOSA AND RUGOSA HYBRIDS}

(Prices as follows unless otherwise noted)

\begin{tabular}{|c|c|c|c|}
\hline a as 101 & & $\begin{array}{ll}\text { Per } & 10 \\
\$ 5 & 00\end{array}$ & $\begin{array}{r}\text { Per } 100 \\
\$ 45 \quad 00\end{array}$ \\
\hline E. Carman & C. F. Worth & $\begin{array}{l}\text { New Century } \\
\text { Nova Zembla }\end{array}$ & \\
\hline Coubert & Iad. Geo. Bruant & $\begin{array}{ll}\text { Rugosa } & \begin{array}{l}\text { Rubra, } \\
\text { Per } 10\end{array}\end{array}$ & Per 100 \\
\hline
\end{tabular}

WICHURIANA OR MEMORIAL

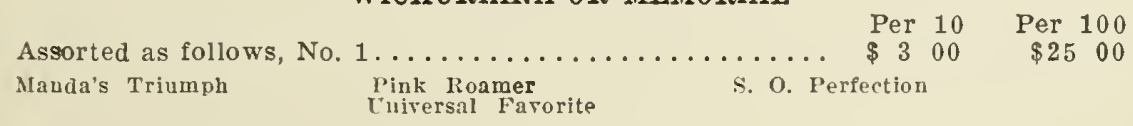




\section{BABY RAMBLERS}

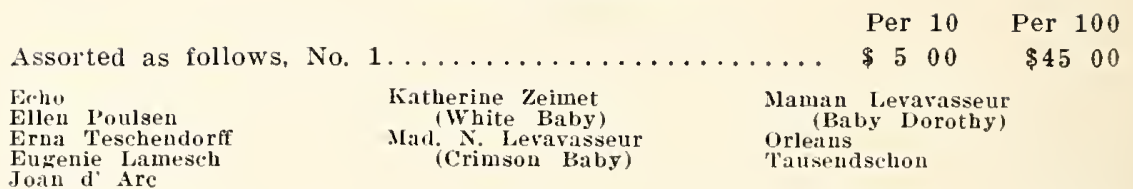

$\begin{array}{rrrr}\text { Per } & 10 & \text { Per } 100 \\ \$ 3 & 50 & \$ 30 & 00\end{array}$

\section{CLIMERNG ROSES}

(Prices as follows unless otherwise noted)

\begin{tabular}{|c|c|}
\hline & Per 10 \\
\hline Assorted as follows, No. $1 \ldots$ & $\ldots \ldots \ldots$ \\
\hline $\begin{array}{l}\text { Baltimore Belle } \\
\text { Blne Rambler } \\
\text { Cilmbing American Beauty at } 30 \mathrm{c} \\
\text { Climbing Baby Rambler } \\
\text { Crimson Rambler at 30c } \\
\text { Dorothy Perkins, white } \\
\text { Dorothy Perkins, pink } \\
\text { Empress of China }\end{array}$ & $\begin{array}{l}\text { Excelsa (Red Dorothy Perkins) } \\
\text { Elower of Fairfield at } 30 \mathrm{c} \\
\text { link lambler } \\
\text { Prairie Queen } \\
\text { Seren Sisters } \\
\text { Tausendschon at 30c } \\
\text { White Rambler } \\
\text { Yellow lambler }\end{array}$ \\
\hline
\end{tabular}

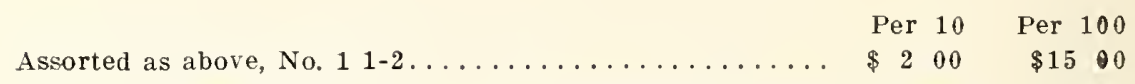

TEA. HYBRID TEA, AND EVERBLOOMING ROSES

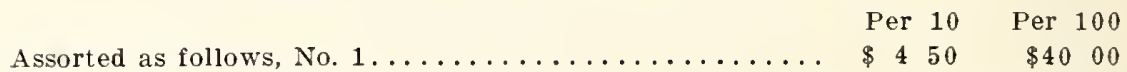

$\begin{array}{ll}\text { Alexander Hill Gray } & \text { General McArthur } \\ \text { American Beauty } & \text { Geo. Dickson } \\ \text { Betty } & \text { Gruss an Teplitz } \\ \text { Bessie Brown } & \text { Jonkeer J. L. Mock } \\ \text { British Queen } & \text { K. A. Victoria } \\ \text { Duchess of Albany } & \text { Killarney, pink } \\ \text { Edward Mawley } & \text { Killaney, white } \\ \text { Etoile de France } & \text { Lady Hillingdon }\end{array}$

Etoile de France

Assorted as above, No. $11-2 \ldots \ldots \ldots \ldots \ldots \ldots \ldots$
La France, pink Madam Caroline Testout Maman Cochet, pink Mrs. Aaron Ward Ophelia

Fed Maman Cochet Sunburst

White Masian Cochet

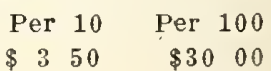

\section{TREE ROSES}

Assorted, No. 1...................... \$15 00

\section{BULBS FOR FALL PLANTING}

Crocus

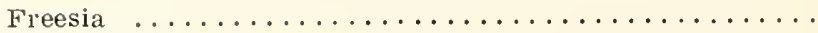

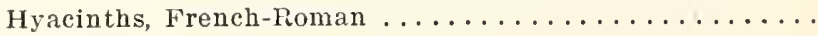

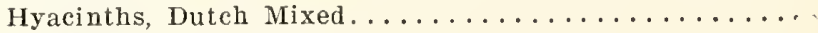

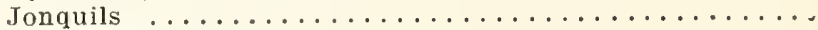

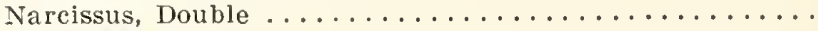

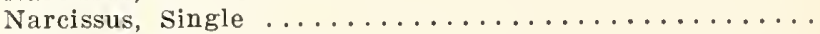

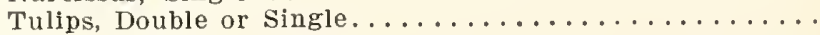

Tulips, Named 


\section{CLIMBING VINES}

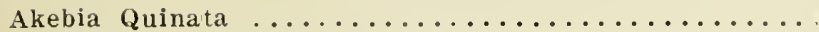

\begin{tabular}{|c|c|c|}
\hline Per & 10 & Per 100 \\
\hline 2 & 50 & $\ldots \ldots$ \\
\hline 1 & 50 & 1200 \\
\hline 1 & 50 & 1200 \\
\hline 2 & 25 & $20 \quad 00$ \\
\hline 1 & 80 & $\ldots$ \\
\hline 2 & 50 & $\ldots$ \\
\hline 2 & 50 & $20 \quad 00$ \\
\hline 2 & 50 & \\
\hline 3 & 75 & 35 \\
\hline
\end{tabular}

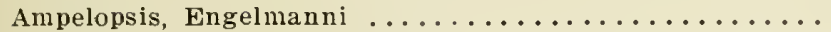

Ampelopsis, Quinquefolia. American Ivy or Virginia Creeper Ampelopsis, Veitchii, Boston Ivy or Japan Creeper......

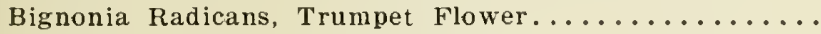

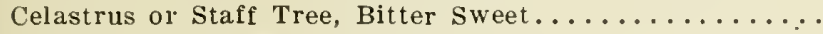

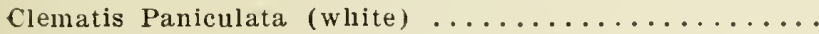

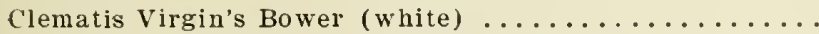

Clematis Large Flowering, as follows

3500

Henryi (white), Jackmanni (purple), Mad. Edward

Andre (red)

Duchess of Edinburgh (white), Ville de Lyon (carmine).

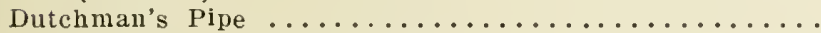

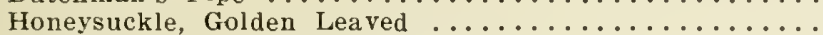

Honeysuckle, Halleana, Hall's Japan.............

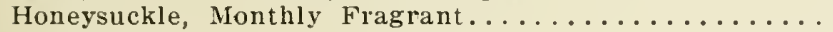

Honeysuckle, Scarlet Trumpet...............

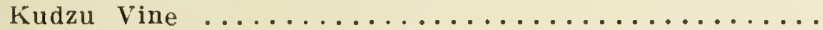

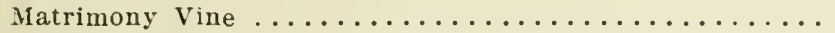

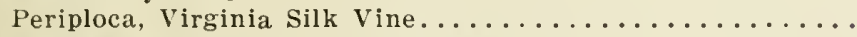

Wistaria, American Purple, Magnifica........... 225

Wistaria, Sinensis, Chinese Blue................... 250

Wistaria, Sinensis, Alba, Chinese White.......... 400

$\ldots \ldots$

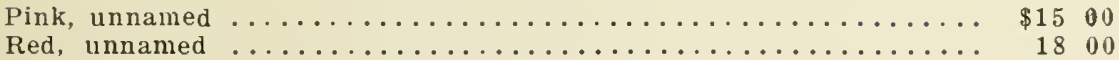

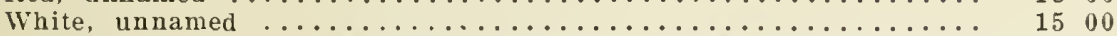

Named Varieties as follows: Adelaide Delachei, Charlemagne, Cythere, Doyenne d' Engheim, Duchesse d' Orleans, Edulis Superba, Glory of Boskoop, Louis Van Houtte, Mme. Chaumy, Marie, Nec Plus Ultra, Reine des Fleurs, Rosea Elegans, Sydonie ........................ \$20.00 Alexander Dumas, Brutus, Canari, Caroline Maguther, Comte de Cuzzy, Delachei, Duchesse de Nemours, Duke of Wellington, Edward Andre, Faust, Festiva Alba, Festiva Maxima, Formosa Rosea, Francois Ortegat, Insignis, Jeanne de Arc, La Tulipe, L' Eclatante, L' Elegante, L' Indespensable, Mme. Crousse, Mme. Forel, Nivea Plenissima, Pres. Roosevelt, Prince Imperial, Prolifera Tricolor, Rubra Triomphans, Solfatare, Victoire Modeste, Zoe

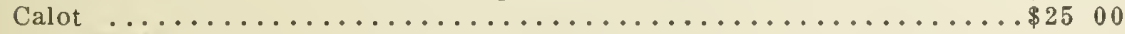
Gen. Millahon, Mme. de Verneville, Monsieur Krelage, Purpurea Superba,

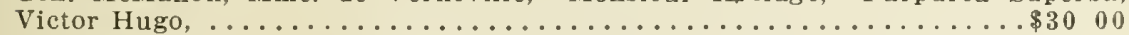
Albatre, La Perle, La Rosiere, Marie Lemoine, Triomphe de L' Exposition de

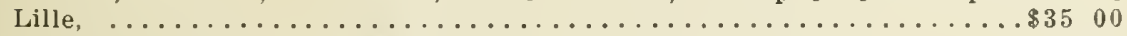
Asa Gray, Felix Crousse, Monsieur Jules Elie, Rose d' Amour....... \$40 00 Adolph Rousseau, Livingstone, Louis Renault................\$50 00

\section{SEEDS}

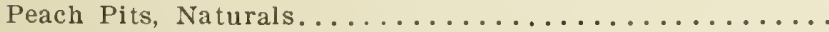

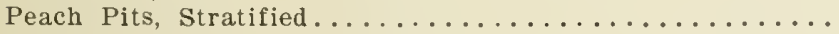

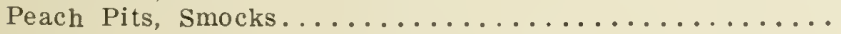

$\$ 500$ per bushel 600 per bushel 400 per bushel 


\section{HARDY PERENNIALS}

Achillea, (The Pearl)

\begin{tabular}{|c|c|c|c|}
\hline Per & 10 & Per & 100 \\
\hline$\$ 1$ & 25 & $\$ 10$ & 00 \\
\hline 1 & 40 & $\ldots$ & . \\
\hline 1 & 25 & 10 & 00 \\
\hline 1 & 25 & 10 & 00 \\
\hline 1 & 00 & $\ldots$ & . \\
\hline 1 & 00 & & 00 \\
\hline 1 & 25 & 10 & 00 \\
\hline 3 & 50 & $\ldots$ & . \\
\hline 1 & 25 & $\cdots$ & • \\
\hline 1 & 50 & $\ldots$ & - \\
\hline 1 & 25 & 10 & 0 \\
\hline 1 & 00 & 8 & 0 \\
\hline 1 & 50 & 12 & 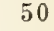 \\
\hline 1 & 25 & 10 & 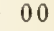 \\
\hline 1 & 25 & 10 & 00 \\
\hline & 70 & 5 & 0 \\
\hline & 80 & 6 & 0 \\
\hline 1 & 40 & 12 & 0 \\
\hline 1 & 20 & 10 & 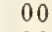 \\
\hline 1 & 75 & 15 & 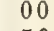 \\
\hline 1 & 50 & 12 & 5 \\
\hline 1 & 20 & 10 & 0 \\
\hline 1 & 00 & $\delta$ & 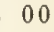 \\
\hline 1 & 25 & 10 & \\
\hline 1 & 40 & 12 & \\
\hline 1 & 25 & 10 & 0 \\
\hline 1 & 25 & 10 & 0 \\
\hline
\end{tabular}

Aquilegia, (Columbine) white or yellow...........

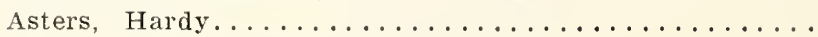

Chrysanthemums, hardy separate colors............

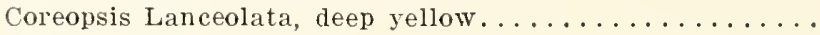

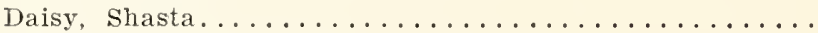

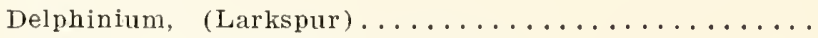

Dielytra Spectabilis (Bleeding Heart)............

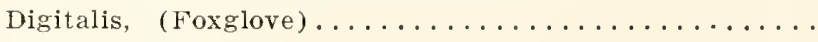

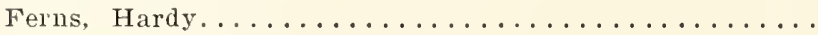

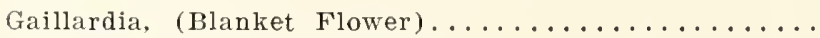

Golden Glow, (Rudbeckia).................

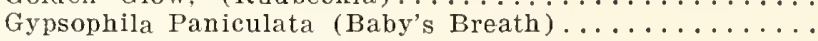

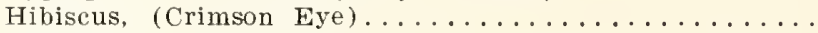

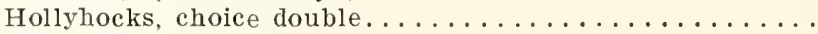

Iris, Liberty, mixed...................

Iris, Liberty, separate colors. . . . . . . . . . . .

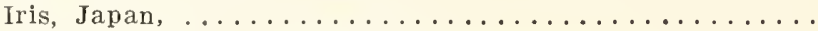

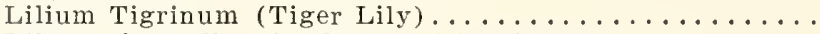

Lily of the Valley, in clumps of 10 pips each.........

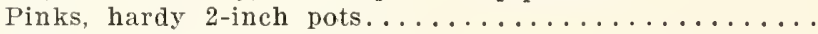

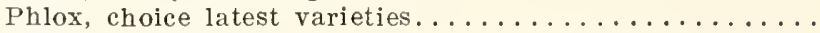

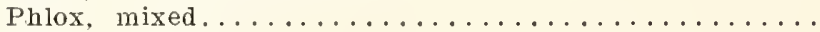

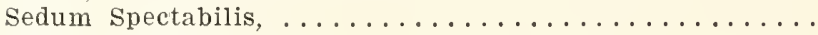

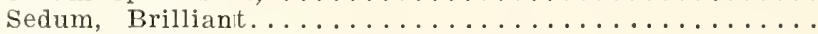

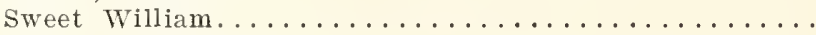

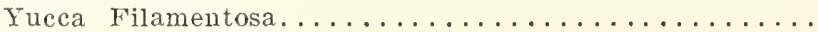

\section{EVERGREENS-Transplanted}

All evergreens are handled in the most careful manner, but we recommend digging with a ball of earth and burlapping. Our price for this work is $35 \mathrm{c}$ for 3-4 feet, $25 \mathrm{c}$ for $2-3$ feet, $18 \mathrm{c}$ for 18 to 24 inches, and $12 \mathrm{c}$ for 12 to 18 inches.

\begin{tabular}{|c|c|c|}
\hline & Per 10 & Per 100 \\
\hline Arbor Vitae, American, 2 to 3 feet. & & $\cdots$ \\
\hline Arbor Vitae, American, 18 to 24 inches............ & 240 & 22 \\
\hline Arbor Vitae, American, 12 to 18 inches.......... & 175 & 15 \\
\hline rbor Vitae, Compacta, 12 to 18 inches............. & 400 & \\
\hline rbor Vitae, Peabody's Golden, 2 to 3 feet. . . . . . . . . . . & 750 & \\
\hline 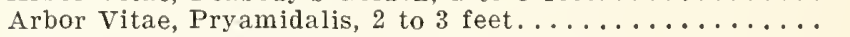 & 750 & \\
\hline Arbor Vitae, Pryamidalis, 18 to 24 inches............ & 500 & \\
\hline Arbor Vitae, Pryamidalis, 12 to 18 inches........... & 400 & \\
\hline Arbor Vitae, Siberian, 18 to 24 inches............. & 400 & \\
\hline bor Vitae, Siberian, 12 to 18 inches............ & 300 & \\
\hline 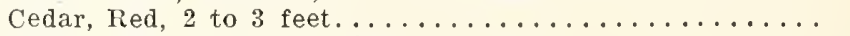 & 500 & \\
\hline 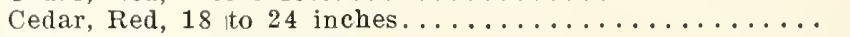 & 400 & \\
\hline , Red, 12 to 18 inches.... & 300 & \\
\hline$\ldots \ldots \ldots \ldots \ldots \ldots$ & 600 & \\
\hline$\ldots \ldots \ldots \ldots \ldots$ & 450 & \\
\hline$\ldots \ldots \ldots \ldots \ldots \ldots$ & 300 & \\
\hline$\ldots \ldots \ldots \ldots \ldots \ldots \ldots$ & 200 & \\
\hline r, Concolor, 3 to 4 & 1000 & \\
\hline Concolor, 2 to & 0 & \\
\hline
\end{tabular}




\section{EVRRGREENS-Continued}

Juniper, Irish, 2 to 3 feet................

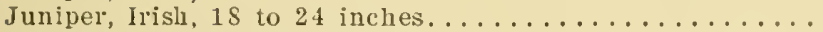

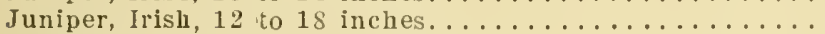

Juniper, Schotti, 5 to 6 feet..................

Juniper, Swedish, 18 to 24 inches.

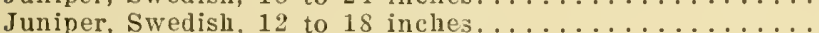

Piue, Austrian or Black, 3 to 4 feet...............

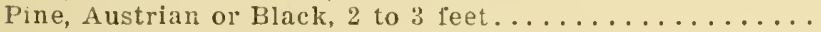

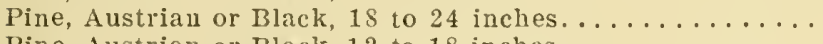

Pine, Austrian or Black, 12 to 18 inches............

Pille, Bull or Pollderosa, 3 to 4 feet.............

Pine, Bull or Ponderosa, 2 to 3 feet..............

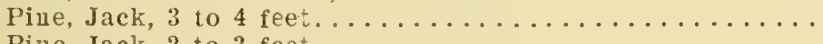

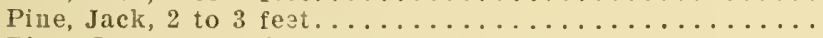

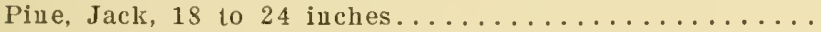

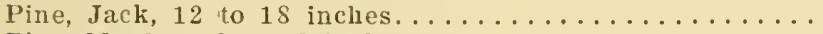

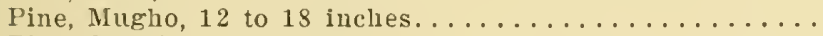

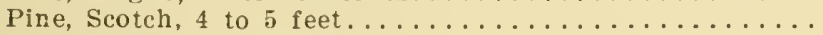

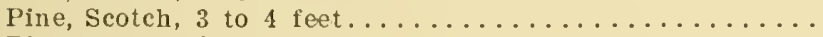

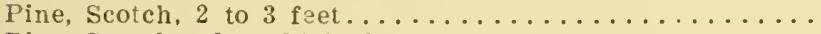

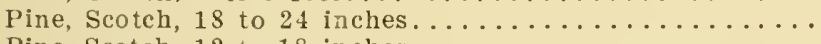

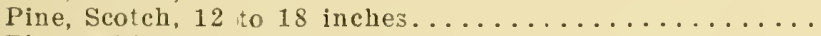

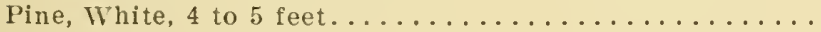

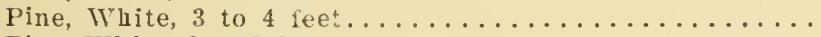

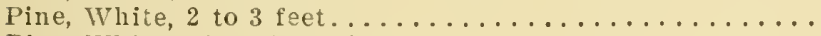

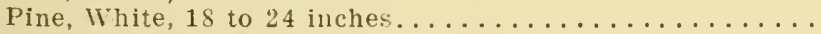

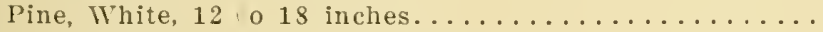

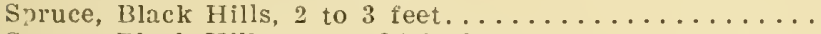

Spruce, Black Hills, 18 to 24 inches..............

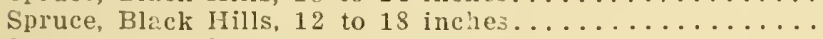

Spruce, Colorado Blue, 3 to 4 feet..............

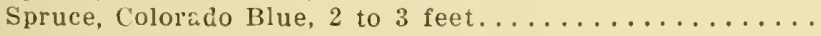

Spruce, Colorado Blue, 18 to 24 inches.............

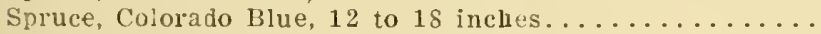

Spruce, Colorado Green, 3 to 4 feet...............

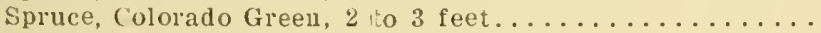

Spruce, Colorado Green, 18 to 24 inches............

Spruce, Colorado Green, 12 to 18 inches............

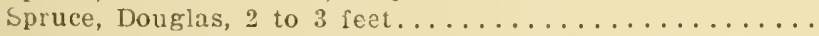

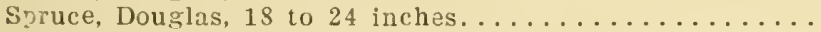

Spruce, Koster's Blue, Grafted. Choice, 5 to 6 feet, B. \& B.

Spruce, Kcsier's Blue, Graftel, Choice, 4 to 5 feet, B. \& B..

Spruce, Kosier's Blue, Grafted, (hoice, 3 1-2 to 4 feet,

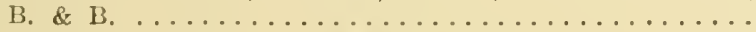

Spruce, liuster's Blue, Grafted, Choice, 3 to 3 1-2 feet, B. \& B. . . . . . . . . . . . . . . . .

\begin{tabular}{|c|c|c|}
\hline Per & 10 & Per 100 \\
\hline 6 & 00 & $\ldots$ \\
\hline 5 & 00 & $\ldots$ \\
\hline 3 & 50 & $\cdots$ \\
\hline 50 & 00 & $\cdots$ \\
\hline 5 & 00 & \\
\hline 3 & 50 & $\ldots$ \\
\hline 5 & 00 & $\cdots$ \\
\hline 4 & 00 & $\cdots$ \\
\hline 3 & 00 & $\cdots$ \\
\hline 2 & $\begin{array}{l}50 \\
50\end{array}$ & $\cdots$ \\
\hline 3 & 50 & $\ldots$ \\
\hline 4 & 00 & $\ldots$ \\
\hline 3 & 50 & $\ldots$ \\
\hline 2 & 50 & $\ldots$ \\
\hline 2 & 00 & $\ldots$ \\
\hline 10 & 00 & $\cdots$ \\
\hline 6 & 00 & $\cdots$ \\
\hline 5 & 00 & $\cdots$ \\
\hline 4 & 00 & $\cdots$ \\
\hline $\begin{array}{l}2 \\
2\end{array}$ & $\begin{array}{l}50 \\
00\end{array}$ & $\ldots$ \\
\hline 5 & 00 & $\ldots$ \\
\hline 4 & 00 & $\ldots$ \\
\hline 3 & 50 & 30 \\
\hline 3 & 00 & 25 \\
\hline 1 & 75 & 15 \\
\hline 5 & 50 & 50 \\
\hline 4 & 00 & 35 \\
\hline 3 & 0.9 & 25 \\
\hline 25 & 00 & $\cdots$ \\
\hline 20 & 00 & $\cdots$ \\
\hline 15 & 00 & $\ldots$ \\
\hline 10 & 00 & $\ldots$ \\
\hline 15 & 00 & $\ldots$ \\
\hline 12 & 50 & $\ldots$ \\
\hline 9 & 00 & . \\
\hline 5 & 00 & . \\
\hline$?$ & 50 & \\
\hline 10 & 00 & \\
\hline 3 & 00 & \\
\hline
\end{tabular}

$60 \quad 00$

$50 \quad 00$

Spruce, Koster's Blue, Grafted, Chcice, $21-2$ to 3 feet,

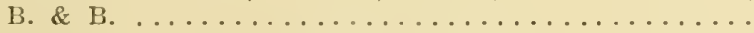

Spruce, Koster's Blue, Grafted, Choice, 2 to 2 1-2 feet, B. \& B.

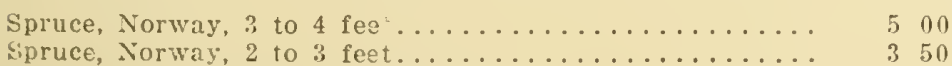

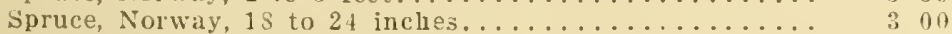

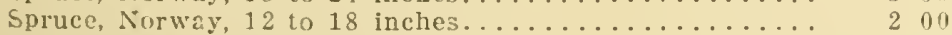

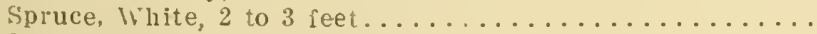

Spruce, White, 18 to 24 inches.

spruce, White, 12 to $1 \mathrm{~S}$ inzles. 


\section{NURSERYMEN'S SUPPIIES}

NOTE-Supplies of all kinds are subject to an advance in price without notice. BOX CLAMP-Lever style, strongest, simplest box clamp we have

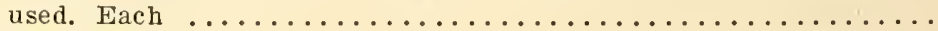

BOX STRAPS-Made of thin sheet steel, cut into strips averaging 10 inches long and 3-4 inch wide. Strong, tough and pliable, Per pound

BURLAP-New, 40 inches wide. Per yard................ 15

COLLATING BOOKS-To collate orders. Per book, postpaid.......

DELIVERY CASH BOOKS-Have ruled spaces for name of customers, amount of order, amount collected, etc., holds 133 accounts. Per

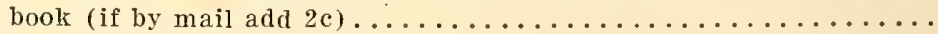

DIBBLES-Made of tooled steel, just the thing for planting grafts,

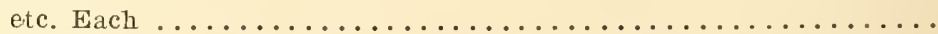

GRAFTING THREAD-Unwaxed. Per ball (if by mail, add $2 \mathrm{c}$ )..... Waxed. One ball will wind about 3,000 grafts (weight 6 oz. per

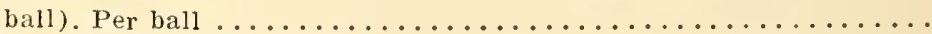

KNIVES, GRAFTING-Keen Kutter, square point (if by mail, add 3c). Each . . . . . . . . . . . . . . . . . . . . .

KNIVES PRUNING-Keen Kutter, fine for light pruning. Each, if by

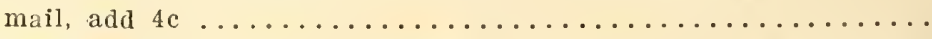

LABELS-Put up 1,000 in a package. Iron wired, 3 1-2 inch plain.

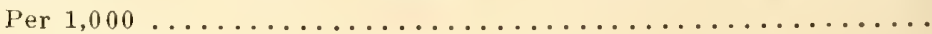

Copper wired, 3 1-2 inch plain. Per $1,000 \ldots \ldots \ldots \ldots \ldots \ldots \ldots$

Pierced, iron wired, 3 1-2 inch plain. Per $1,000 \ldots \ldots \ldots \ldots \ldots \ldots$

Moss-Bales $14 \times 18 \times 22$, Wired. Per bale.................

NEEDLES - For sewing burlap. 5-inch curved, best. Per dozen........

ORDER BOOKS-Contain 50 order sheets, also duplicates of different color. Our name does not appear on these blanks. Each.........

PAPER - To line boxes. Comes in $70 \mathrm{lb}$. rolls, 40 inches wide. Per pound

PRUNING SHEARS-California pattern, 9 inches, volute spring. Each SEWING TWINE-For burlapping, in skeins. Per pound.......... SHINGLE TOW-Baled, average about 100 lbs. to the bale. Per 100

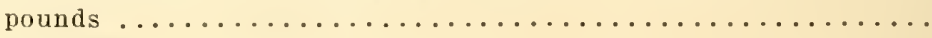

SISAL-2 ply, for tying bales. Per pound................

SPADES-Ames make. (WVe have this pattern made to our order.) It weighs only 7 pounds, is strapped full length, non-tapering. Each. .

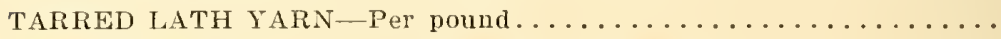

TUBE ROPE-4-ply for tying trees for storage, put up in $5 \mathrm{~b}$. balls,

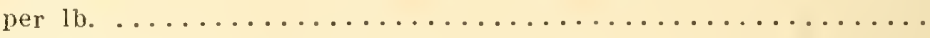

WOOL TWINE-3-ply, for tying trees; put up in 1 pound balls. Per pound 